Subscriber access provided by Yale University Library

\title{
Synthesis of Ni Nanoparticles by Fs Laser \\ Ablation in Liquids: Structure and Sizing
}

David Muñetón Arboleda, Jesica María Jose Santillán, Luis Joaquín Mendoza Herrera, Marcela B. Fernández van Raap, Pedro Mendoza Zélis, Diego Muraca, Daniel Schinca, and Lucía Scaffardi

J. Phys. Chem. C, Just Accepted Manuscript • DOI: 10.1021/acs.jpcc.5b03124 • Publication Date (Web): 13 May 2015

Downloaded from http://pubs.acs.org on May 15, 2015

\section{Just Accepted}

"Just Accepted" manuscripts have been peer-reviewed and accepted for publication. They are posted online prior to technical editing, formatting for publication and author proofing. The American Chemical Society provides "Just Accepted" as a free service to the research community to expedite the dissemination of scientific material as soon as possible after acceptance. "Just Accepted" manuscripts appear in full in PDF format accompanied by an HTML abstract. "Just Accepted" manuscripts have been fully peer reviewed, but should not be considered the official version of record. They are accessible to all readers and citable by the Digital Object Identifier (DOI®). "Just Accepted" is an optional service offered to authors. Therefore, the "Just Accepted" Web site may not include all articles that will be published in the journal. After a manuscript is technically edited and formatted, it will be removed from the "Just Accepted" Web site and published as an ASAP article. Note that technical editing may introduce minor changes to the manuscript text and/or graphics which could affect content, and all legal disclaimers and ethical guidelines that apply to the journal pertain. ACS cannot be held responsible for errors or consequences arising from the use of information contained in these "Just Accepted" manuscripts. 


\title{
Synthesis of Ni Nanoparticles by Fs Laser Ablation in Liquids: Structure and Sizing
}

David Muñetón Arboleda, ${ }^{\dagger}$ Jesica M. J. Santillán, ${ }^{\dagger},+$ Luis J. Mendoza Herrera, ${ }^{\dagger}$ Marcela B. Fernández van Raap, ${ }^{\ddagger, l}$ Pedro Mendoza Zélis, ${ }^{\ddagger l,}, \S$ Diego Muraca, ${ }^{\#}$ Daniel C. Schinca, ${ }^{\dagger, \S}$ and Lucía B. Scaffardi $*, \dagger, \S$

${ }^{\dagger}$ Centro de Investigaciones Ópticas (CIOp), CONICET La Plata - CIC, Camino Centenario y 506, 1897 Gonnet, La Plata, Argentina

${ }^{\ddagger}$ Facultad de Ciencias Exactas y Naturales, Universidad Nacional de Catamarca, Av. Belgrano 300, 4700 San Fernando del Valle de Catamarca, Catamarca, Argentina

${ }^{\ddagger}$ Instituto de Física La Plata (IFLP), CONICET - UNLP, Calle 49 y 115, 1900 La Plata, Buenos Aires, Argentina

"Departamento de Física, Facultad de Ciencias Exactas, Universidad Nacional de La Plata, Calle 49 y 115, 1900 La Plata, Buenos Aires, Argentina

${ }^{\S}$ Departamento de Ciencias Básicas, Facultad de Ingeniería, Universidad Nacional de La Plata, Calle 115 y 49, 1900 La Plata, Buenos Aires, Argentina

"Instituto de Física "Gleb Wataghin" (IFGW), Universidade Estadual de Campinas, Rua Sérgio Buarque de Holanda 777, Cidade Universitária Zeferino Vaz Barão Geraldo CEP 13083-859, Campinas, São Paulo, Brasil

\section{Supporting Information}

\begin{abstract}
Synthesis of nickel (Ni) nanoparticles (NPs) suspensions was performed using a 120 femtosecond (fs) pulse laser to ablate a Ni solid target in n-heptane and water. Analysis of structure, configuration and sizing was carried out using different independent techniques such as Optical Extinction Spectroscopy (OES), Atomic Force Microscopy (AFM), Transmission Electron Microscopy (TEM) and Electron Diffraction (ED), which yield interrelated information.

AFM microscopy allows determining the spherical shape and size distribution of the NPs in the obtained colloids while TEM provides knowledge about shape, structure and size distribution. ED allows identification of the different metal and metal oxide composition as well as their crystallographic phase. On the other hand, OES gives
\end{abstract}


information related to size distribution, structure, configuration and composition. Interpretation of these spectra is based on Mie theory which, in turn, depends on $\mathrm{Ni}$ dielectric function. For NPs radii smaller than $3 \mathrm{~nm}$, size-dependent free and bound electron contributions to the dielectric function must be considered. To account for the full size span, complete Mie expansion was used for optical extinction cross-section calculations. A theoretical analysis of the dependence of plasmon resonance of bare core and core-shell Ni NPs with core size and shell thickness provide insight about their spectroscopic features.

For n-heptane, species like bare core Ni and hollow Ni NPs are found in the colloid, being the latter reported for the first time in this work. Instead, for water, the colloid contains hollow nickel NPs and nickel oxide in different core-shell configurations: $\mathrm{Ni}-\mathrm{NiO}$ and $\mathrm{NiO}-\mathrm{Ni}$, being the latter also reported for the first time in this paper. In both cases, size distribution agrees with that derived from TEM and AFM analysis. The formation of the oxide species is discussed in terms of oxidation-reduction processes during ablation. Possible mechanisms for the formation of hollow species are proposed.

KEYWORDS: Ni Nanoparticles, Femtosecond Laser Ablation, Optical Extinction Spectroscopy, Size-Dependent Dielectric Function, Optical Properties, Plasmon Resonance

\section{INTRODUCTION}

Metal NPs studies became the focus of intense research owing to their unusual properties compared with bulk metal, which convey many potential applications in different science and technology fields. ${ }^{1}$ The control of physical, chemical and 
biological properties in the nanometric scale is based on the ability to manipulate the size and shape of NPs. ${ }^{2-7}$

In recent years, Ni NPs have attracted scientific interest because of their extensive prospects in catalysts, ${ }^{8,9}$ conducting pastes, ${ }^{10}$ information storage, ${ }^{8}$ large-scale batteries, ${ }^{11}$ magnetic behavior, ${ }^{12-15}$ enhanced optical properties, ${ }^{16}$ and biomedicine. ${ }^{17}$

Several works on Ni NPs generation by laser ablation using different pulsed laser regimes and different media have appeared in the literature in the last years. Liu et al. studied the morphological and structural properties of Ni NPs generated by 500 fs pulse laser ablation in low pressure background gas. ${ }^{18}$ They determined that by limiting the laser fluence below the threshold of plasma formation, large particles may be avoided. Besides, by varying the background gases, Ni-NiO core-shell spheres were obtained. Amoruso et al. used 300 fs laser pulses focused on a Ni target in vacuum to study the expansion dynamics of the ablated species by fast photography and optical emission spectroscopy. ${ }^{19}$ Dudoitis et al. generated Ni NPs in argon and air using a $\mathrm{Nd}$ :YAG laser working in two different pulsed regimes, $15 \mathrm{~ns}$ and $10 \mathrm{ps}$, and describing the influence of laser intensity and thermal capacities on the electron and lattice temperature. $^{20}$

On the other hand, generation of Ni NPs via laser ablation in liquids has been recently reported by Jaleh et al., who worked with a high repetition Nd:YAG laser of 240 ns pulse width for ablating a pure Ni plate immersed in methanol with PVP as stabilizer agent, studying their localized surface plasmon via optical spectroscopy. ${ }^{21}$ Jung and Choi have worked with a Q-switched Nd:YAG laser at its fundamental wavelength to ablate $\mathrm{Ni}$ target in different solvents and have studied their final crystallographic phase, concluding that it is related to the thermodynamic factors of the laser-induced plasma plume and to the specific heat of the solvents. ${ }^{22}$ Musaev et al. 
fabricated colloids of $\mathrm{Ni}$ in water by laser ablation with a nitrogen laser of $10 \mathrm{~ns}$ pulse duration and determined its composition using X-ray diffraction (XD). ${ }^{23}$

However, there are no works reporting on the fabrication and characterization of colloids obtained using fs-regime laser ablation of $\mathrm{Ni}$ in liquids. In this work we analyze the characteristics of structure (bare core or core-shell), configuration, number density and size distribution of NPs generated when a fs pulse laser is used to ablate a Ni solid target in n-heptane and water. Optical extinction was used to obtain the spectroscopic characteristics of freshly fabricated suspensions. This technique is appropriate for this type of samples since it probes a statistically relevant number of particles (about $10^{10}$ to $10^{11}$ particles) and can yield qualitative and quantitative information about particle size distribution and number density. Extinction spectra were modeled using Mie theory ${ }^{24}$ through an appropriate modification of the bulk complex dielectric function, taking into account a size-dependent corrective term for each free and bound electron contribution. We theoretically explore the dependence of extinction spectrum with size for bare core and core-shell Ni NPs. This spectrum is dominated by plasmon resonance whose wavelength is dependent on size, shell thickness and surrounding media.

Here we report for the first time the synthesis of Ni NPs suspensions using a 120 fs pulse laser to ablate a Ni solid target in n-heptane and water, the presence of NiO-Ni core-shell and hollow Ni NPs in these colloids and propose a possible mechanism for the formation of different species present in the obtained suspensions. AFM, TEM and ED analyses independently performed on the colloid samples yielded size and structural results that agree with those obtained by OES. 


\section{EXPERIMENTAL SECTION}

Nickel colloidal suspension was fabricated by fs pulse laser ablation. The target sample used to carry out these experiments was a $1 \mathrm{~mm}$ thick solid disk of high purity grade nickel, immersed in a vessel filled with $4 \mathrm{~cm}^{3}$ of $\mathrm{n}$-heptane or milli-Q water. This solvent volume created a liquid column of $1 \mathrm{~cm}$ height over the Ni disk. Laser ablation was performed using a Ti:Sapphire (Ti:Sa) chirped pulsed amplification (CPA) system from Spectra Physics, emitting pulses of $120 \mathrm{fs}$ with at $1 \mathrm{kHz}$ repetition rate centred at $800 \mathrm{~nm}$ wavelength. The maximum output energy was $1 \mathrm{~mJ}$ per pulse, but it could be attenuated using a classical waveplate-polarizer system. A $5 \mathrm{~cm}$ focal length lens was used to focus the laser beam on the target disk surface. The pulse energy used in this experiment was $100 \mu \mathrm{J}$. Considering the focal length of the focusing lens and the input beam diameter, the corresponding fluence value was $90 \mathrm{~J} / \mathrm{cm}^{2}$. The experimental setup chosen to produce nickel NPs in liquid media is shown in Figure 1.

The sample was moved using a XY motorized micrometric stage. The movement was programmed so that the laser impinged always in different points of the sample. In this way, ablation was performed always from a fresh surface of the sample. This process lasted $9 \mathrm{~min}$, after which the large number of NPs generated in the surfactant free suspension produced a greyish colour in n-heptane or whitish in water. 


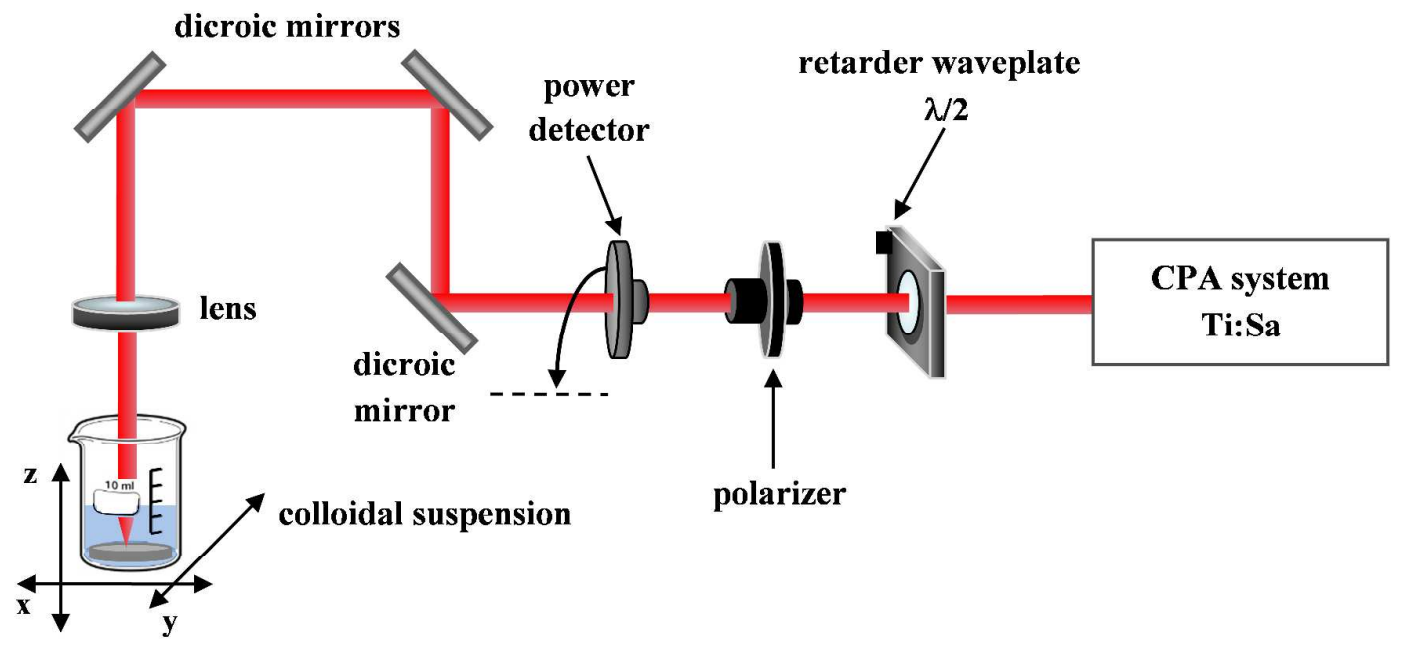

Figure 1. Schematic diagram of experimental setup for the fabrication of nickel colloidal suspension.

AFM images were recorded in air, at room temperature, and at a scan rate of $1 \mathrm{~Hz}$, using standard semicontact mode of an NT-MDT Solver Pro microscope. Rectangular shaped semi contact-mode cantilevers coated with $\mathrm{Al}$ on the reflex side, having a spring constant of $37-58 \mathrm{~N} / \mathrm{m}$ and holding a pyramidal silicon nitride tips of tip curvature radius of $6 \mathrm{~nm}$ were used for topographical measurements. APPNANO ACTA and ACLA probes at resonant frequencies of $273 \mathrm{kHz}$ and $166 \mathrm{kHz}$ were used for samples in $n$-heptane and in water respectively. AFM provides a three-dimensional surface profile. Lateral dimension are distorted by tip-sample convolution effects. However, high $\mathrm{z}$ accuracy is obtained when height measurements with minimum scanning vertical step equal to $0.012 \mathrm{~nm}$ are recorded. For these measurements, part of the as-prepared colloidal suspension was sonicated and diluted to $1 / 1000(\mathrm{v} / \mathrm{v})$ in n-heptane and 1/100 (v/v) in water. A drop of such diluted sample was placed on a freshly cleaved muscovite mica sheet V-1 grade (SPI Supplies) and dried for $12 \mathrm{~h}$ at room temperature. The analysis of mica sheet measurement indicated an average roughness of $0.0612 \mathrm{~nm}$. 
Microscopy images and electron diffraction patterns were performed with a TEM-MSC (JEOL 2100, acceleration voltage $200 \mathrm{KV}$ ) at Brazilian Nanotechnology National Laboratory (LNNano). The as-prepared Ni was diluted in n-heptane or mili-Q water and sonicated during $15 \mathrm{~min}$. Samples were obtained by drying the solventdispersed NPs on a carbon coated copper grids followed by plasma cleaning to avoid possible organic residuals. Images of the samples on a single-tilt sample holder were recorded/captured using a TV Gatan ES500W and a CCD (TVips-16MP) camera.

OES was implemented by means of a Shimadzu spectrophotometer from $250 \mathrm{~nm}$ to $1100 \mathrm{~nm}$ wavelength range. Optical absorption measurement of the colloidal suspension in each liquid was performed $5 \mathrm{~min}$ after fabrication. This in situ measurement prevents possible NPs coalescence and provides reliable statistics.

\section{THEORETICAL BACKGROUND FOR EXTINCTION SPECTRA CALCULATION}

As it is well known, $\mathrm{Ni}$ is a ferromagnetic metal. In accordance to the analysis made by Rosensweig, ${ }^{25}$ the frequency dependence of monodomain magnetic NPs complex susceptibility follows a Lorentzian law with frequency and relaxation time: $\chi(\omega)=\frac{\chi_{0}}{1+i \omega \tau}$, where $\chi_{0}$ is the static field susceptibility, and $\tau$ is the magnetic relaxation time. For the case of single domain Ni NPs, the value of $\tau$ is typically in the range $10^{-2}-10^{-6} \mathrm{~s},{ }^{26}$ which, for visible frequencies $\left(10^{14}-10^{15} \mathrm{~Hz}\right)$ yield $\chi(\omega)$ very close to zero. Under these conditions, the relative permeability is unity $\left(\mu_{r}=1\right)$ allowing the NP extinction to be correctly described by Mie theory ${ }^{24}$ for non magnetic NPs.

In general, light extinction is the sum of absorption and scattering processes that can be studied using the mentioned theory. For the general case of coated spherical 
particles with core radius $R$ and outer radius $R^{\prime}$, the extinction cross section can be expressed as:

$$
C_{\text {ext }}=\frac{2 \pi}{k^{2}} \operatorname{Re}\left\{\sum_{n=1}^{\infty}(2 n+1)\left(a_{n}+b_{n}\right)\right\}
$$

where $a_{n}$ and $b_{n}$ are the scattering coefficients which depend on the first, second and third order spherical Bessel functions. The argument of these functions depends on the core and shell size parameters and their refractive indices (see Supporting Information). Sometimes, the so called extinction efficiency, defined as $Q_{\text {ext }}=C_{\text {ext }} / \pi R^{\prime 2}$, is used for easiness of comparison.

The refractive index of materials is related to the dielectric function. For the case of metals, the experimental bulk complex dielectric function $\left(\varepsilon_{\text {bulk }}(\omega)\right)$ may be modeled as an additive contribution of free and bound electrons. Free electron contribution is usually written based on the Drude model, while bound electron contribution may be written considering interband transitions:

$$
\varepsilon_{\text {bulk }}(\omega)=\varepsilon_{\text {free }}(\omega)+\varepsilon_{\text {bound }}(\omega)=\varepsilon^{\prime}(\omega)+i \varepsilon^{\prime \prime}(\omega)
$$

where free electrons contribution can be written as:

$$
\varepsilon_{\text {free }}(\omega)=1-\frac{\omega_{p}^{2}}{\omega^{2}+i \gamma_{\text {free }} \omega}
$$

and bound electron contribution as:

$$
\varepsilon_{\text {bound }}(\omega)=\varepsilon_{\text {bulk }}(\omega)-\left(1-\frac{\omega_{p}^{2}}{\omega^{2}+i \gamma_{\text {free }} \omega}\right)
$$


In these expressions, $\omega_{p}$ is the bulk plasma frequency and $\gamma_{\text {free }}$ is the damping constant of the electron oscillatory movement. The latter represents the damping of electron oscillation due to different processes: electron-electron collisions, electron-ion collisions and electron-phonon collisions. These processes are characterized by the mean time between two successive collisions and are taken into account altogether in the $\gamma_{\text {free }}$ value, since they cannot be specifically separated within the parameterization of the bulk dielectric function.

When the size of metal NPs is smaller than the electron mean free path, the dielectric function must be size corrected. The new expression may be written adding size corrective terms for free and bound electrons to the experimental bulk dielectric function:

$$
\varepsilon_{\text {size }}(\omega, R)=\varepsilon_{\text {bulk }}(\omega)+\delta \varepsilon_{\text {free }}(\omega, R)+\delta \varepsilon_{\text {bound }}(\omega, R)
$$

where

$$
\delta \varepsilon_{\text {free }}(\omega, R)=\omega_{p}^{2}\left(\frac{1}{\omega^{2}+i \gamma_{\text {free }} \omega}-\frac{1}{\omega^{2}+i \gamma_{\text {free }} \omega+i C \omega \frac{v_{F}}{R}}\right)
$$

and

$$
\delta \varepsilon_{\text {bound }}(\omega, R)=-e^{-\left(\frac{R}{R_{o}}\right)}\left(\varepsilon_{\text {bulk }}(\omega)-1+\frac{\omega_{p}^{2}}{\omega^{2}+i \gamma_{\text {free }} \omega}\right)
$$

In equation (6) $v_{F}$ is the Fermi velocity, $C$ is a constant that depends on the material and on the electron scattering processes at the particle boundary. It has a mean value of $0.8 .^{27}$ 
In equation (7), the factor $e^{-\left(\frac{R}{R_{0}}\right)}$ is related to the larger energy level separation (and consequently a minor density of states) when the particle goes to smaller sizes while the number of atoms decreases. ${ }^{28}$

Both size corrections $\delta \varepsilon_{\text {free }}(\omega, R)$ and $\delta \varepsilon_{\text {bound }}(\omega, R)$ in equation (5) depend on Fermi velocity and Drude model parameters $\omega_{p}$ and $\gamma_{\text {free }}$. The procedure to determine the two latter parameters was described in detail in the work by Mendoza Herrera et al. ${ }^{29}$ Briefly, two linear parametric equations involving the real and imaginary parts of $\varepsilon_{\text {free }}(\omega)$ can be derived from equation (3). When these equations are plotted with the experimental frequency as a parameter, linear regressions may be obtained only for large wavelengths where Drude model is dominant. The slopes of these regressions yield $\omega_{p}$ and $\gamma_{\text {free }}$. Using the experimental values for the complex refractive index of $\mathrm{Ni}$ reported by Ordal et al. in the wavelength range from $670 \mathrm{~nm}$ to $80000 \mathrm{~nm},{ }^{30}$ $\omega_{p}=(6.41 \pm 0.12) \times 10^{15} \frac{1}{\mathrm{~s}}$ and $\gamma_{\text {free }}=(3.16 \pm 0.35) \times 10^{13} \frac{1}{\mathrm{~s}}$ were determined

Figure 2 shows the real and imaginary parts of $\mathrm{Ni}$ size-dependent dielectric function versus wavelength for different radii considering the bulk refractive index data given by Rakić et al., ${ }^{31}$ which coincides with Ordal et al. in the NIR-mid IR but extends the data to wavelengths smaller than $670 \mathrm{~nm}$. Theoretical calculations were performed based on equations (5) to (7), using $\omega_{p}$ and $\gamma_{\text {free }}$ mentioned above, $C=0.8$, $v_{F}=2.8 \times 10^{14} \frac{\mathrm{nm}}{\mathrm{s}}, 32$ and $R_{O}=0.35 \mathrm{~nm}$. 


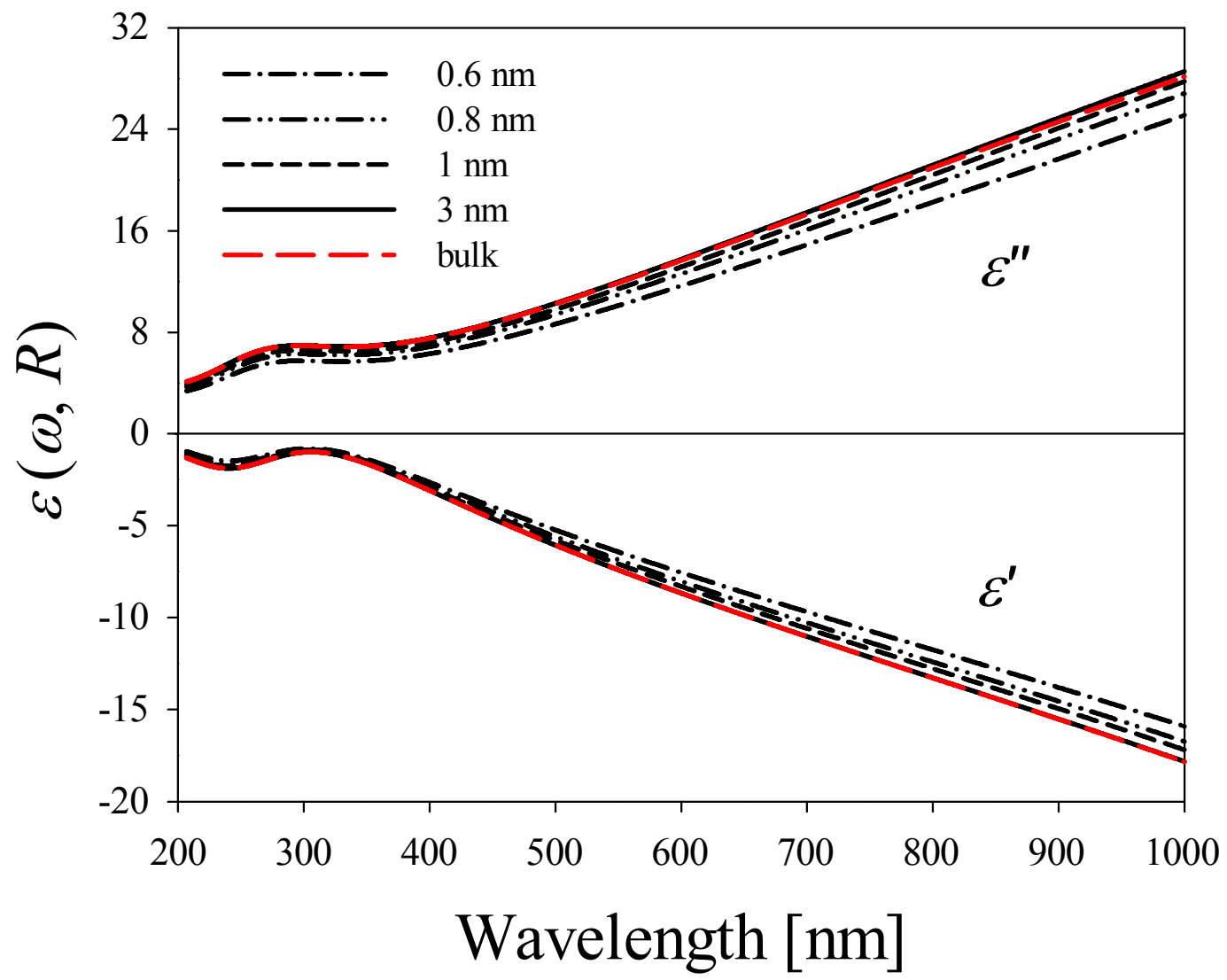

Figure 2. Calculated values of real and imaginary parts of Ni size-dependent dielectric function vs wavelength for different radii using equations (5) to (7).

The experimental bulk dielectric function is represented by the curve in dashed line. The curve for $3 \mathrm{~nm}$ radius (full line) is almost superimposed to that for bulk, indicating that for larger radii $(R>3 \mathrm{~nm})$ size corrections are negligible. However, for sizes smaller than $3 \mathrm{~nm}$, curves depart from the bulk dielectric function in the considered wavelength range between $200 \mathrm{~nm}$ and $1000 \mathrm{~nm}$.

Figure 3 shows the extinction efficiency $Q_{\text {ext }}$ of bare Ni NPs in water for radii between $2 \mathrm{~nm}$ and $60 \mathrm{~nm}$, plotted as a function of wavelength using equation (1). A plasmon resonance for $7 \mathrm{~nm}$ radius can be observed as a small shoulder on the extinction curve. This resonance is progressively redshifted up to $550 \mathrm{~nm}$ and increases in intensity as the radius increases from $7 \mathrm{~nm}$ to $60 \mathrm{~nm}$. 


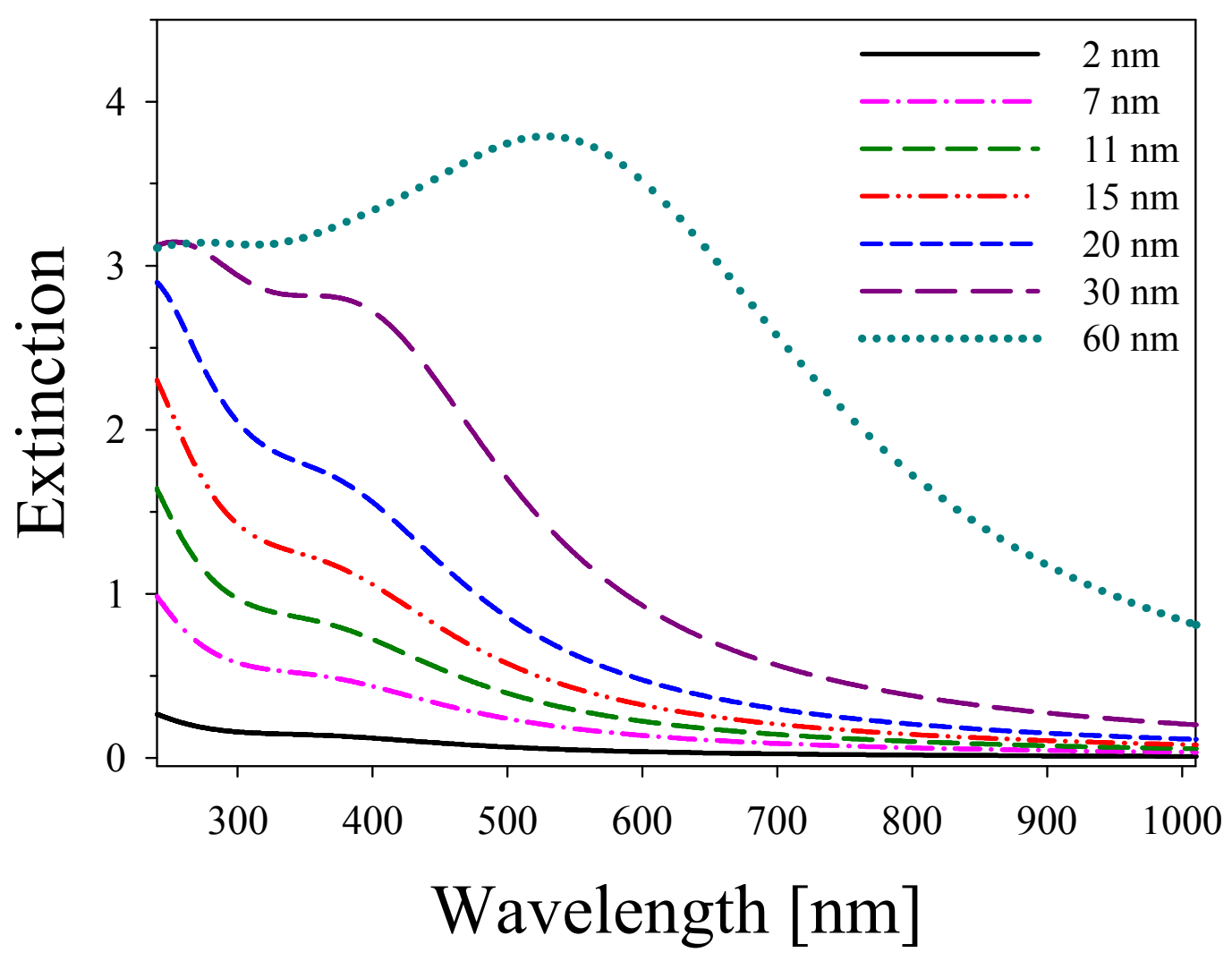

Figure 3. Extinction spectra of Ni bare core NPs immersed in water. Plasmon resonance is redshifted and increases in intensity as the NPs radius increases.

A similar behavior in plasmon resonance redshift is observed when extinction is calculated for bare Ni NPs in n-heptane.

If the particles are core-shell type, the extinction efficiency depends on the inner radius $R$ and the outer radius $R^{\prime}$ (see Supporting Information). Figure 4 shows the extinction spectra of core-shell air-Ni NPs in water for a core radius $R=10 \mathrm{~nm}$ and different percentages of shell thicknesses. For this particle size, the shape of the spectra is still due mainly to absorption, since scattering contribution is negligible. 


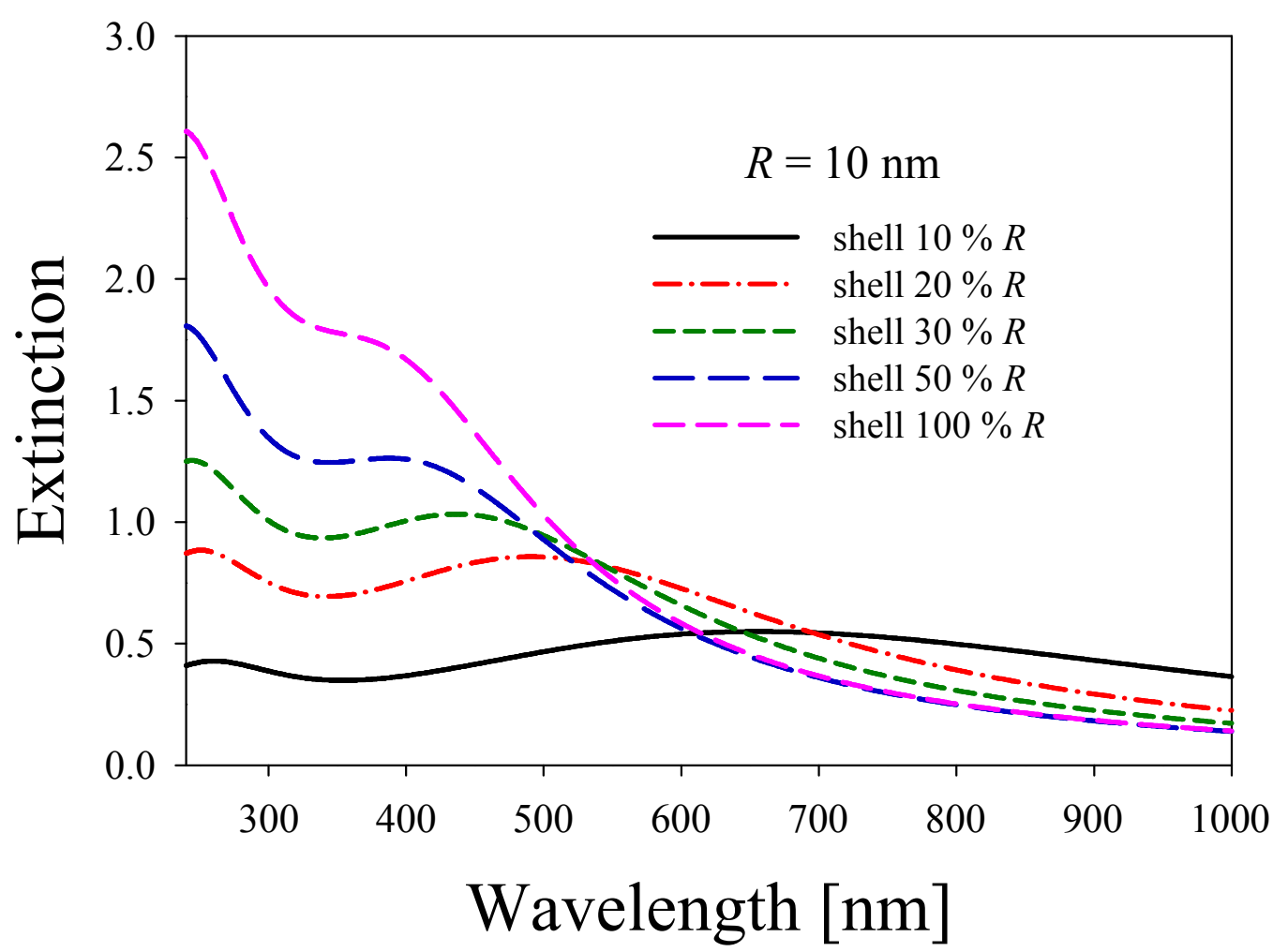

Figure 4. Extinction spectra for core-shell air-Ni NPs in water for core radius $R=10 \mathrm{~nm}$ and different shell thicknesses.

The plasmon resonance that appears around $400 \mathrm{~nm}$ for $100 \%$ shell thickness redshifts as the shell thickness decreases, falling in intensity and broadening.

When the Ni target is ablated in water, oxidation-reduction processes may occur. ${ }^{18,33}$ In this case, it is necessary to consider the possibility of generating core-shell NPs of the type Ni-NiO and NiO-Ni. Under these conditions, it is important to calculate their extinction spectra, together with its dependence on shell thickness. Figure 5a shows the extinction spectra of core-shell Ni-NiO NPs in water for a core radius $R=10 \mathrm{~nm}$ and different percentages of shell thicknesses. As the NiO shell increases, the plasmon resonance at $400 \mathrm{~nm}$ is slightly redshifted while it decreases in intensity. Figure $5 \mathrm{~b}$ shows the extinction spectra of core-shell NiO-Ni for the same core radius and several shell thickness. In this case, the plasmon resonance is strongly redshifted as 
the shell thickness decreases, producing a broadening of the resonance. This behavior is similar to that of dielectric core-noble metal shell NP. ${ }^{34}$ In both cases, dielectric function of $\mathrm{NiO}$ was taken from Mahmoud et al. ${ }^{35}$

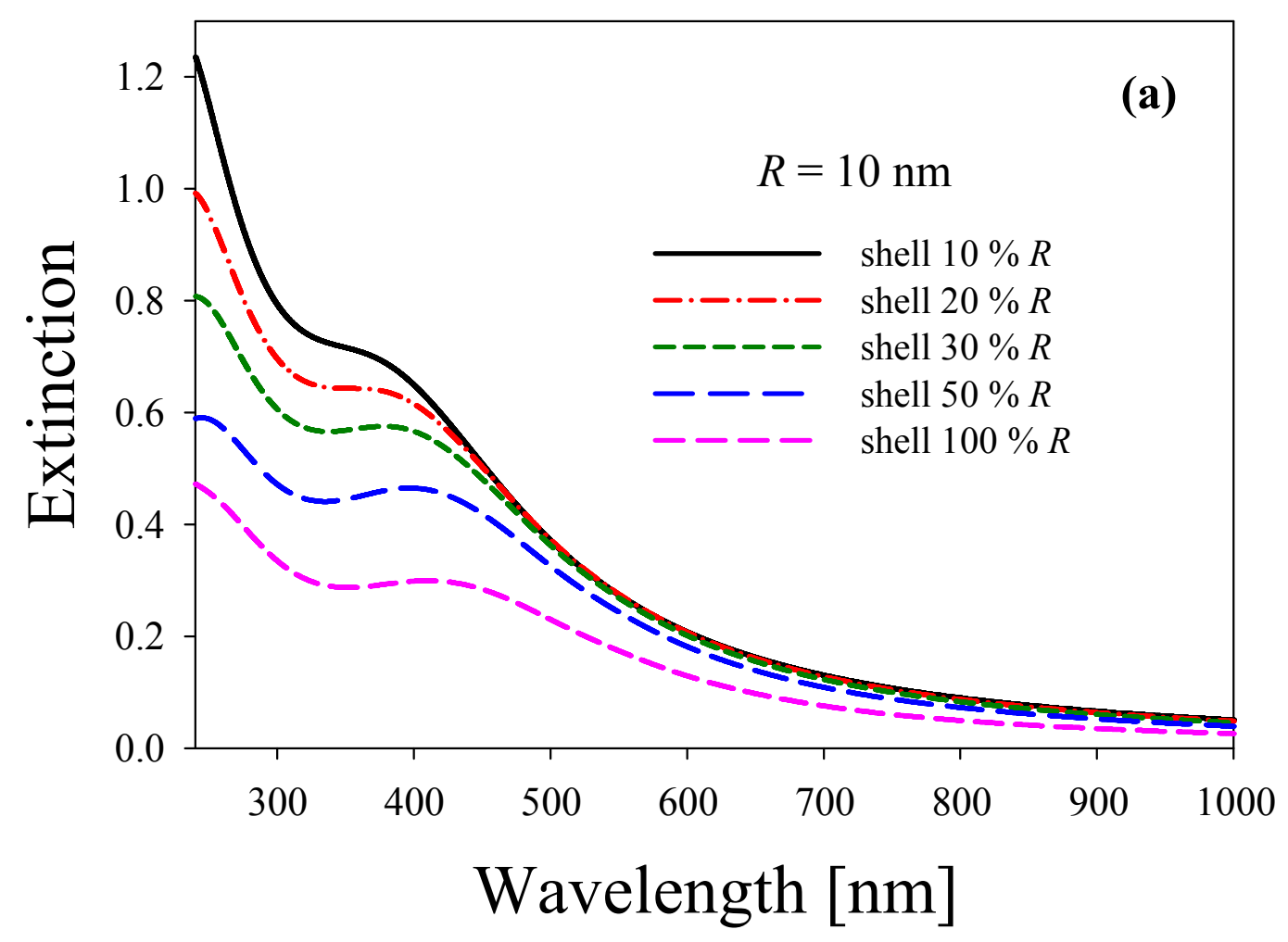




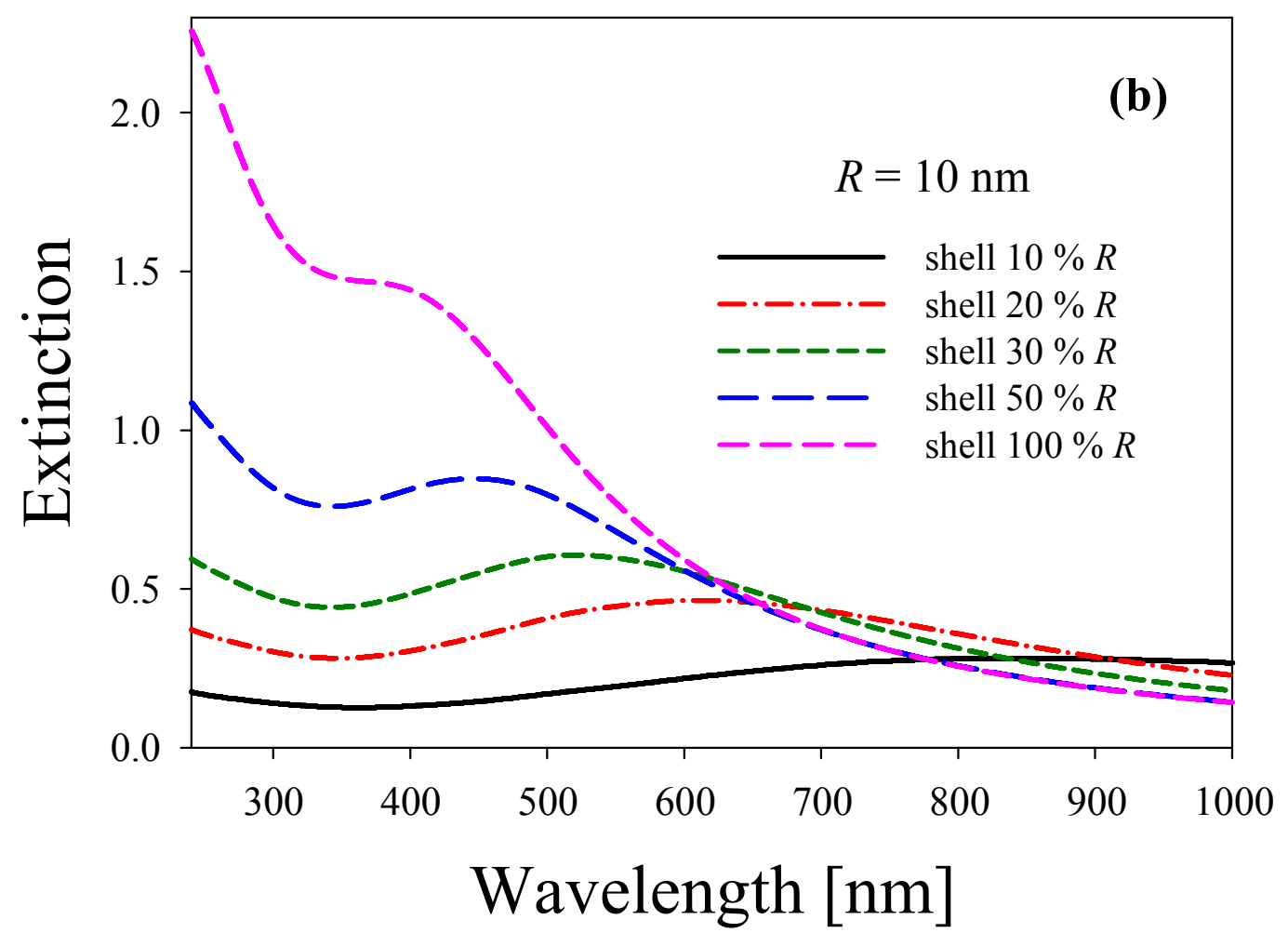

Figure 5: Optical extinction spectra calculated for (a) Ni-NiO and (b) NiO-Ni core-shell NPs in water, for core radius $R=10 \mathrm{~nm}$ and different shell thicknesses.

If the surrounding medium is n-heptane instead of water, a similar behavior in plasmon tuning with shell thickness is observed.

\section{EXPERIMENTAL RESULTS AND DISCUSSION}

\section{a. Ni Colloidal Suspension in n-Heptane}

Experimental extinction spectra of Ni colloidal suspension obtained by fs pulse laser ablation in n-heptane were performed immediately after preparation. Figure 6 shows a typical extinction spectrum of Ni NPs suspension in n-heptane normalized at $\lambda=340 \mathrm{~nm}$. Full line represents the experimental curve while dashed-dotted line represents the fit according to the size distribution shown in the inset. The particle 
sizing is represented in it by log-normal size distributions of bare core Ni NPs with modal radii at $2.5 \mathrm{~nm}$ and $10 \mathrm{~nm}$ together with air-Ni core-shell NPs at external modal radii of $6.6 \mathrm{~nm}(R=6 \mathrm{~nm}$; shell $10 \% R), 12 \mathrm{~nm}(R=10 \mathrm{~nm}$; shell $20 \% R)$ and $15.6 \mathrm{~nm}$ $(R=15 \mathrm{~nm} ;$ shell $4 \% R)$.

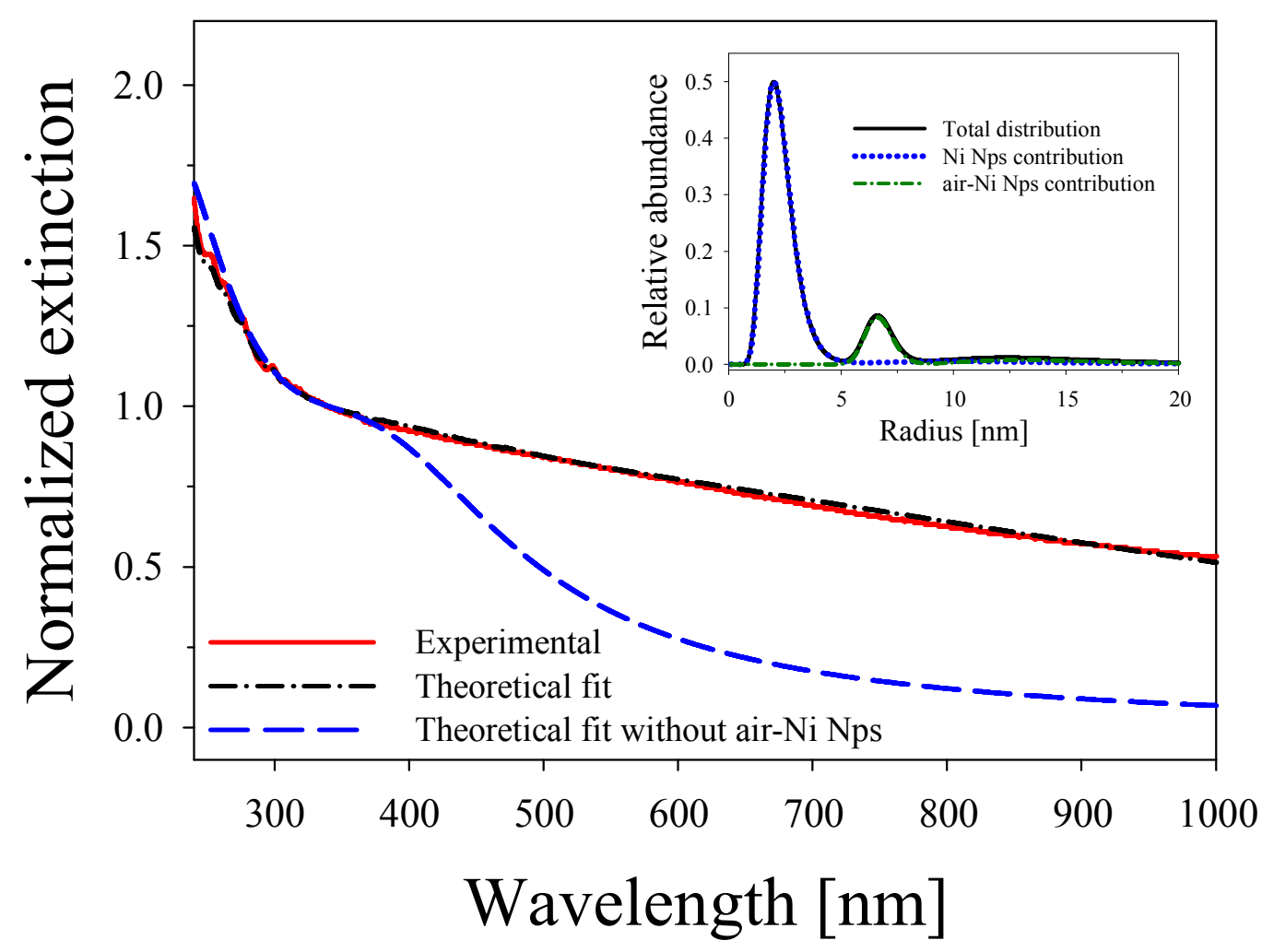

Figure 6. Comparison between experimental (full line) and theoretical (dashed-dot line) extinction spectra of $\mathrm{Ni}$ colloidal suspension in n-heptane. Inset shows bare core and air-Ni relative abundance contributions that fit experimental spectrum. Long dashed line represents calculated spectrum with the same distribution without taking into account air-Ni NPs.

Based on the relative abundance curve shown in the inset of Figure 6 and taking into account the Lambert Beer Law, we can calculate the number density $\left(N_{i}\right)$ of each species present in the colloidal suspension through a factor that relates the experimental normalizing point at a given wavelength with the theoretical normalizing point at the 
same wavelength. The equation relating the number density $N_{i}$ and the relative abundance $\delta_{i}$ (in $\mathrm{cm}^{-2}$ units) of the $i$-th species is $N_{i}=\frac{\alpha \delta_{i}}{\beta L}$, where $\alpha$ is the experimental spectrum normalization factor and $\beta$, the theoretical spectrum normalization factor. This normalization can be carried out for any given wavelength. In our case $\alpha=0.182$ and $\beta=114.95$ for $\lambda=340 \mathrm{~nm}$. In this way, it is possible to determine the number density of bare core Ni and air-Ni NPs of different radii present in the colloidal suspension as shown in Table 1. From OES measurements, the total number of NPs probed by the spectrophotometer beam is approximately $2 \times 10^{10}$. This value shows the high statistics of OES technique.

Table 1. Number density and relative percentage of different species of NPs present in the colloidal suspension in n-heptane

\begin{tabular}{ccc}
\hline Species & Number density $\left[\mathbf{c m}^{-3}\right]$ & $\begin{array}{c}\text { Relative } \\
\text { percentage }\end{array}$ \\
\hline Ni bare core & $1.34 \times 10^{11}$ & $82 \%$ \\
Air-Ni core-shell & $2.95 \times 10^{10}$ & $18 \%$ \\
\hline
\end{tabular}

A key contribution for the accurate fit of the experimental extinction spectrum from $350 \mathrm{~nm}$ onward can be found in the extinction behavior of hollow-type NPs. As shown for the aqueous suspension in Figure 4, the extinction spectrum corresponding to air-Ni NPs for $10 \mathrm{~nm}$ inner radius and Ni thickness smaller than $10 \%$ of core radius shows a very broad resonance centered at about $700 \mathrm{~nm}$. This feature contributes to fit the extinction spectra for $\lambda>350 \mathrm{~nm}$. Calculated extinction without considering the contribution of the air-Ni NPs is represented by the dashed line in Figure 6. It is readily 
seen that the curve fits the experimental spectrum only up to $350 \mathrm{~nm}$, departing noticeably from it for larger wavelengths.

Air-metal core-shell structures have been reported by other authors by ablating specific materials using different kind of pulsed lasers. For example, Desarkar et al. obtained $\mathrm{Zn} / \mathrm{ZnO}$ hollow NPs using a 6 ns Nd:YAG pulsed laser ablation of zinc in liquids. ${ }^{36}$ Yan et al. fabricated hollow Pt and $\mathrm{Al}_{2} \mathrm{O}_{3}$ NPs using a 50 ns pulsed excimer laser for ablating solid $\mathrm{Pt}$ and $\mathrm{Al}$ targets in water. ${ }^{37-39}$ These authors propose that the hollow particles are formed at the boundary of laser-produced bubbles, which provide a preferred nucleation site and diffusion sink for self assembly of the laser-fabricated clusters. Niu et al. obtained hollow NPs of diverse metal oxides and sulfides by laser ablating metal targets in specific liquids, based on the Kirkendall effect after heating with an infrared laser. ${ }^{40}$ More recently, we have found this type of hollow structures using 120 fs pulsed laser ablation of a silver target in water, ${ }^{41}$ apart from other kind of species present in the colloidal suspension. Our results on silver ablation and on $\mathrm{Ni}$ ablation with fs regime, suggest that a similar formation mechanism about laser induced bubbles may produce the observed hollow NPs. Although the relative abundance of Ni hollow NPs in the colloidal suspension is small (about $18 \%$, as indicated by the dash dotted line in inset of Figure 6), their influence on the shape on the extinction spectrum in the Vis-NIR region is noticeable as can be seen in Figure 6 (dashed line curve).

AFM images of single NPs were obtained to characterize shape and size of the $\mathrm{Ni}$ colloidal suspension generated in n-heptane. Figure 7a shows $50 \mu \mathrm{m} \times 50 \mu \mathrm{m}$ scanned area image where small isolated NPs are observed. Height profiles scanned in lines 1 to 5 show NPs with typical external diameters of $15 \mathrm{~nm}, 27 \mathrm{~nm}, 5 \mathrm{~nm}, 22 \mathrm{~nm}$ and $23 \mathrm{~nm}$ respectively, as indicated in Figure $7 \mathrm{~b}$. For these small size values, it is important to consider the roughness of the mica substrate on which the drop was 
deposited. Line 3 in panels (a) and (b) shows the height profile of the mica background across the imaged area. It can be seen that the mean roughness is about one order of magnitude smaller than the smallest recorded particle, so there is a very good signal-tonoise ratio in the nanoparticle's profile. Panel (c) shows the radius histogram of the NPs in the image of panel (a). The histogram can be fitted by two log-normal size distributions centered at modal radius $R_{\mathrm{m} 1}^{\prime}=2 \mathrm{~nm}$ and $R_{\mathrm{m} 2}^{\prime}=7 \mathrm{~nm}$.

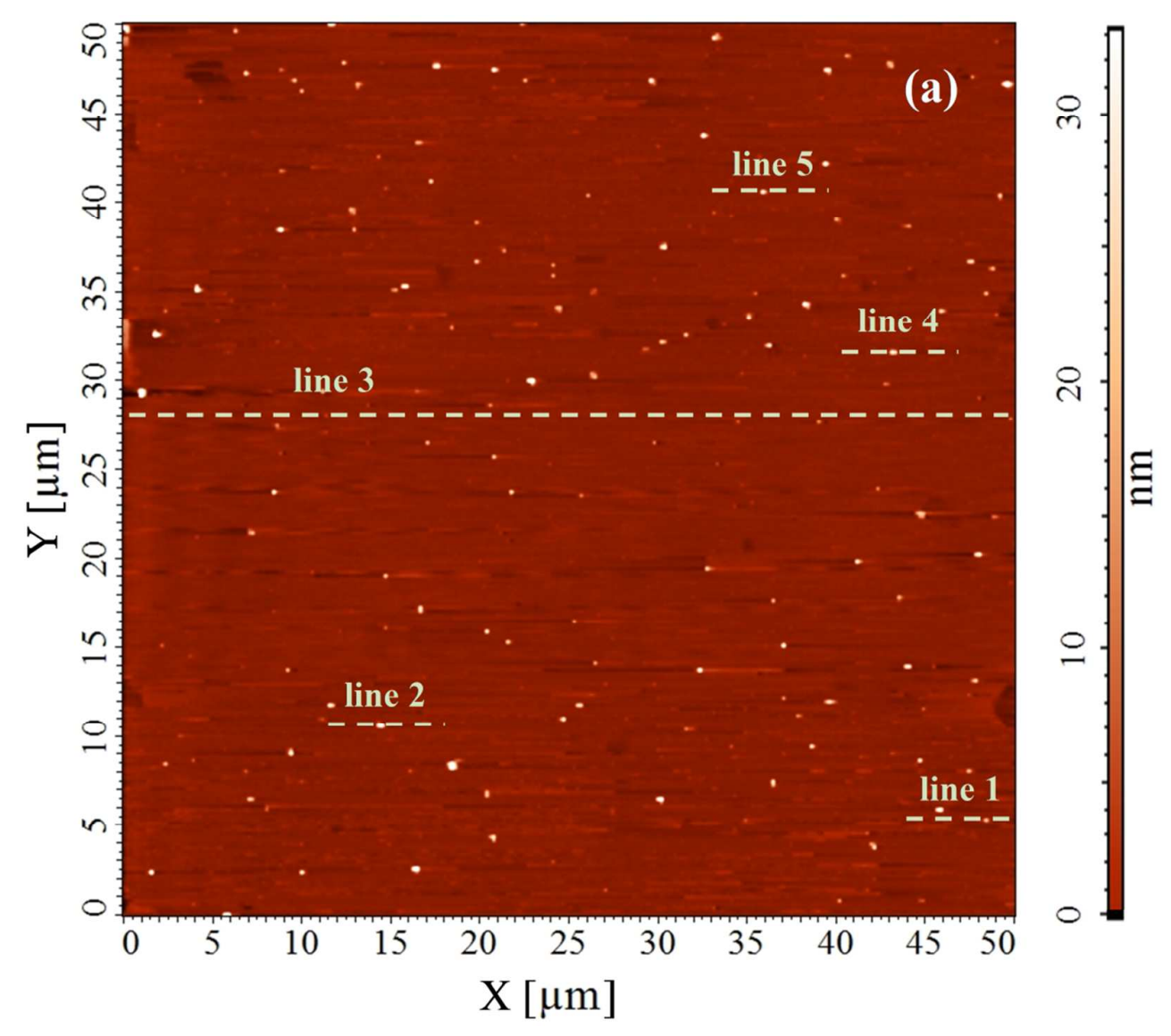



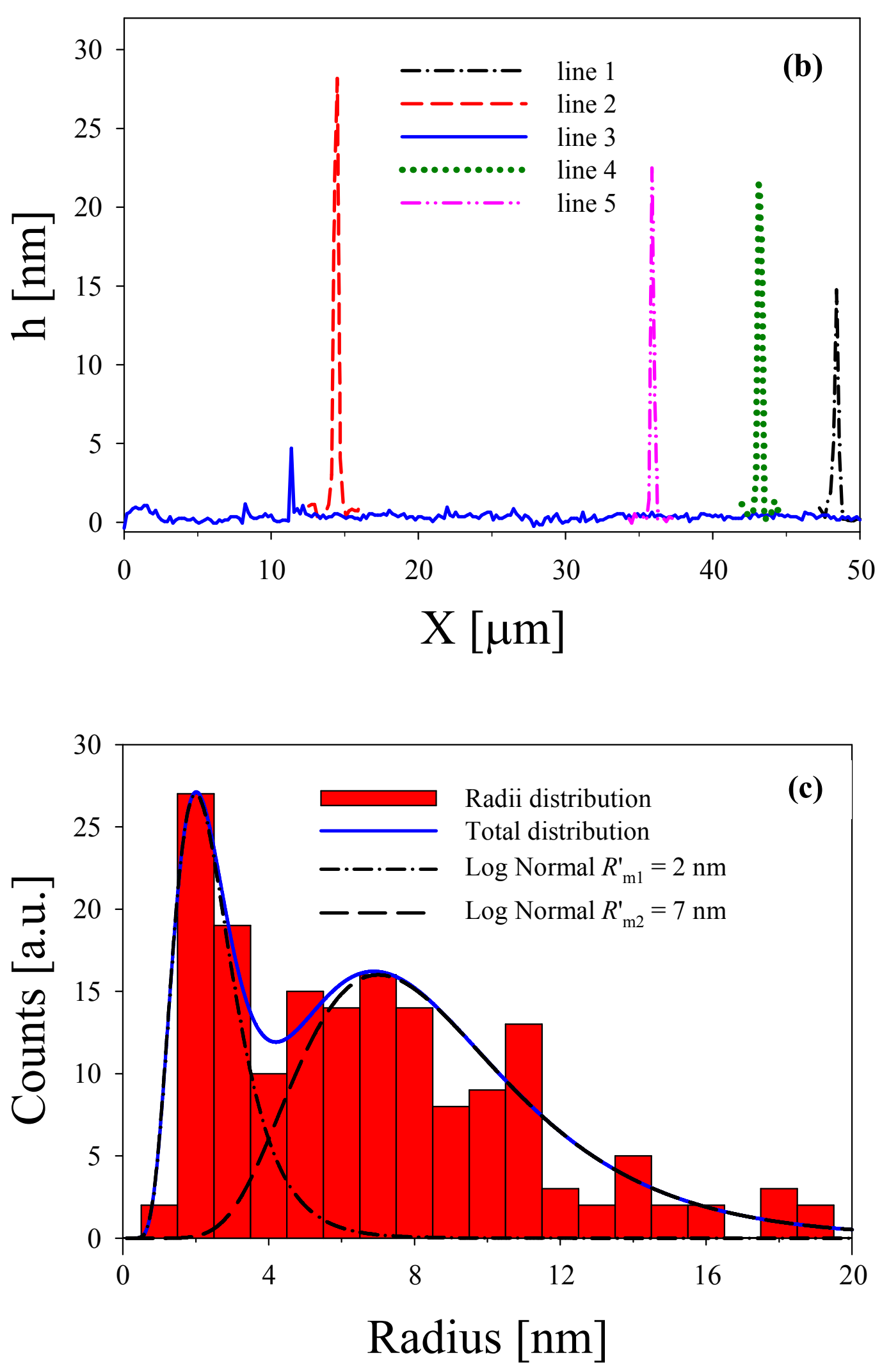
Figure 7. AFM size analysis of the obtained Ni colloidal suspension in n-heptane: (a) image of isolated single Ni NPs; (b) height profiles of selected lines 1 to 5 from panel (a) which correspond to the spherical particles diameter; (c) radius histogram together with two log-normal size distributions at $R_{\mathrm{m} 1}^{\prime}=2 \mathrm{~nm}$ and $R_{\mathrm{m} 2}^{\prime}=7 \mathrm{~nm}$. Full line represents the sum of both distributions.

Although the amount of particles observed in the AFM image is much smaller than that probed by OES, it can be seen that the heights in panel (b) and the histogram in panel (c) of Figure 7 are in good agreement with the external radii distribution determined from the fit of the extinction spectrum of the sample shown in the inset of Figure 6.

To study the morphology of Ni NPs, TEM analysis was carried out. Figure 8 shows TEM images of Ni colloidal suspension fabricated by ablation in n-heptane. Panel (a) shows a panoramic view of isolated bare core and hollow Ni NPs. Panel (b) is an enlargement of a bare core Ni NP from panel (a). Panel (c) is another panoramic view together with an enlargement of an air-Ni NP. Dotted line outlines the limit of the core and shell structure. Panel (d) shows the radius histogram corresponding to a statistics performed over several TEM images. The results can be fitted by two lognormal size distributions which describe the most prominent features of the histogram. 

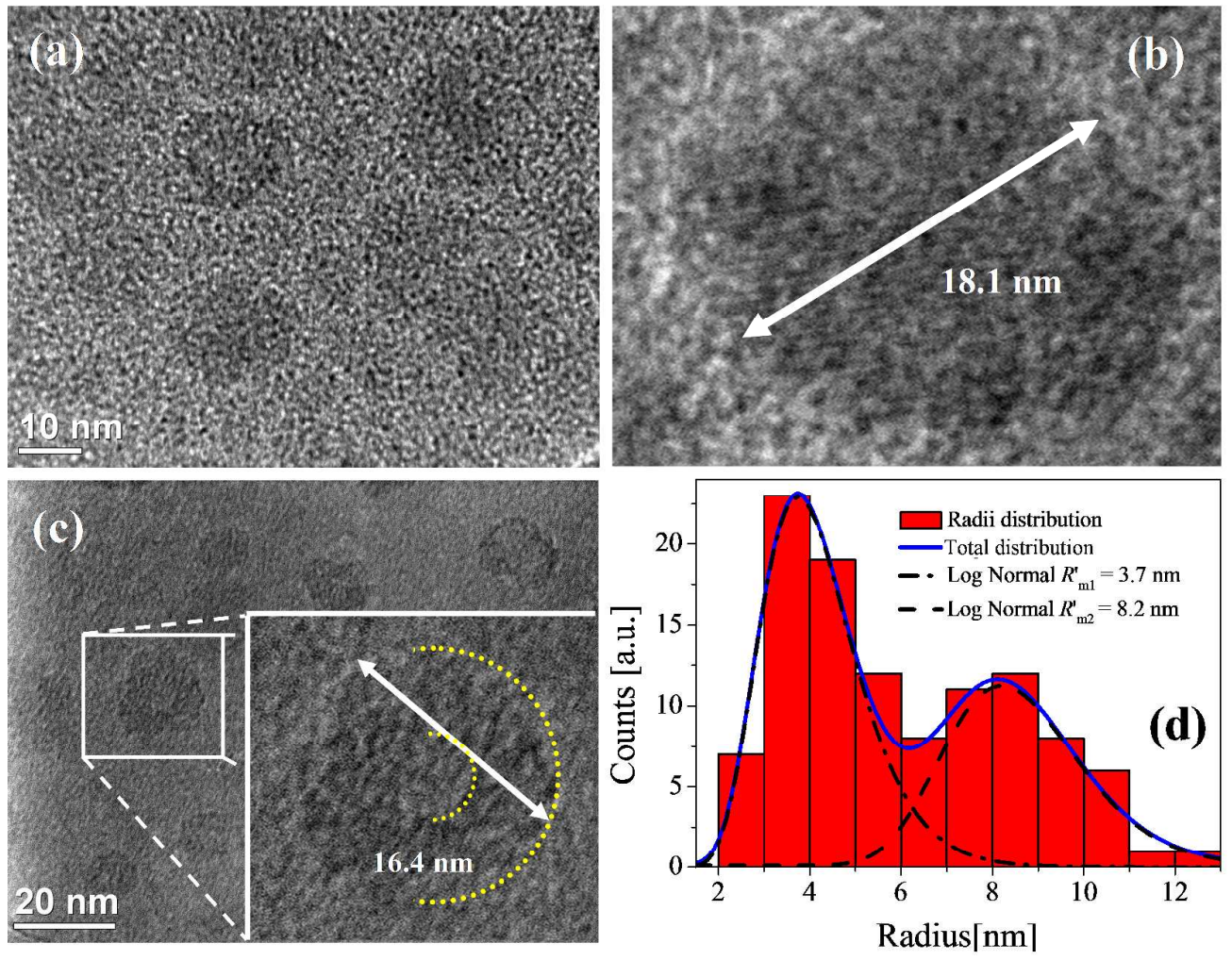

Figure 8. TEM image of NPs present in the Ni colloidal suspension in n-heptane. (a) Panoramic view; (b) single isolated bare core Ni NP; (c) detail of a hollow Ni NP and (d) radius histogram and log-normal size distributions which account for the most prominent features of the histogram.

It is interesting to notice that the sizes arising from TEM analysis (Figure 8) as well as those obtained from AFM (Figure 7) are in agreement with results derived from OES shown in Figure 6. Spherical bare core and core-shell structures can be seen. This fact supports the use of Mie approach for calculating extinction spectra as well as the inclusion of this type of hollow particles for fitting the spectrum.

\section{b. Ni Colloidal Suspension in Water}

Ni colloidal suspensions in water were studied with the same methods as before.

Figure 9 shows a typical extinction spectrum in the range UV-visible-near IR 
normalized at $\lambda=340 \mathrm{~nm}$. Full line represents the experimental extinction curve, while dashed-dotted line is the theoretical fit. In this case, since the solvent is water, some degree of oxidation is surely to occur. Structures and configurations like bare core NiO besides core-shell structures of $\mathrm{Ni}-\mathrm{NiO}, \mathrm{NiO}-\mathrm{Ni}$ as well as air-Ni were considered to be present in the colloidal suspension. The inset shows in full line the total size distribution that yield the optimum fit of the experimental spectrum. Specific size distribution for Ni-NiO NPs (dotted line), air-Ni NPs (dashed-dotted line) and NiO-Ni NPs (dashed line) are also shown. The latter size distribution includes bare core NiO NPs species. It can be noticed that the multimodal size distribution shape is similar to that determined for n-heptane but shifted to larger values of external radii. This fact may be due to the presence of oxide shells around the NPs. Each one of the previously mentioned species influence the extinction spectrum in specific and distinct regions in such a way that the combination of structures, configurations, sizes and relative abundances derived from optimum fit constitute a unique set of fitting parameters. For instance, theoretical calculation without considering air-Ni NPs, will not fit the experimental extinction spectrum for wavelengths larger than $400 \mathrm{~nm}$ (long dashed line in Figure 9). Similarly to the effect shown for n-heptane, the exclusion of this type of particles precludes the correct fit of the spectrum for this wavelength range. Notice that, although the abundance of air-Ni NPs is rather small (see inset), it has a very large effect in the fitting procedure. A similar lack of fitting is observed when the contribution of NiO-Ni NPs is not considered (not shown in Figure 9). For a given species type any small variation in size or relative abundance produce a noticeable change in the shape of the spectrum. 


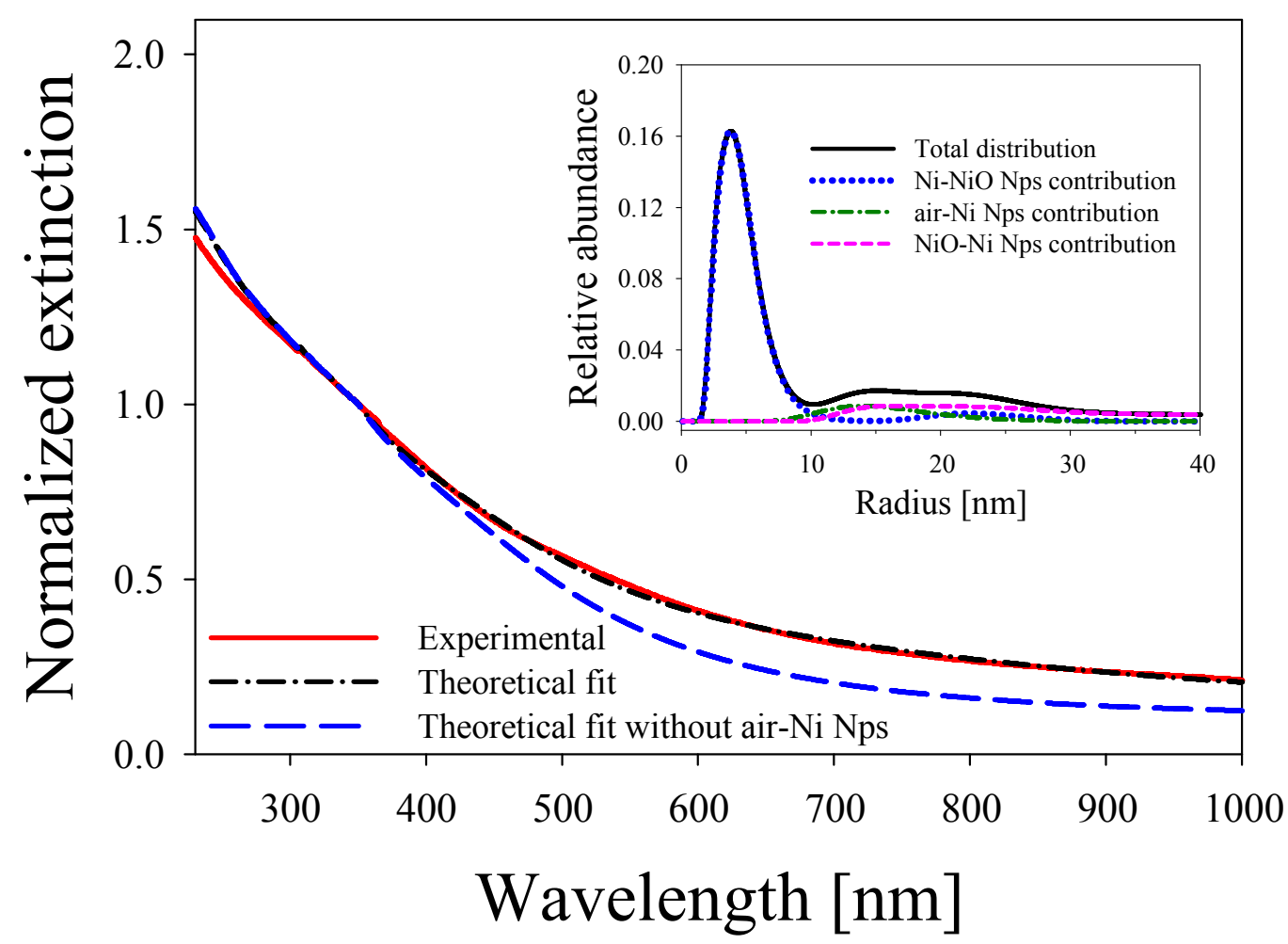

Figure 9. Comparison between experimental (full line) and theoretical (dashed-dots line) extinction spectra of Ni colloidal suspension in water. Inset shows size distribution of $\mathrm{Ni}-\mathrm{NiO}$, air-Ni and NiO-Ni NPs that fits experimental spectrum. Long dashed line represents calculated spectrum with the same distribution without considering air-Ni NPs contribution.

As in the case of n-heptane, OES technique allows to calculate the number density of the different structures and configurations present in the colloidal suspension in water. Considering the relative abundance curve shown in the inset of Figure 9, $\alpha=0.553$ and $\beta=488.323$ for $\lambda=340 \mathrm{~nm}$ it is possible to calculate the number density of Ni-NiO, Air-Ni and NiO-Ni core-shell species present in all sizes in the colloidal suspension fabricated in water. Results are shown in Table 2 
Table 2. Number density and relative percentage of different species of core-shell NPs present in the colloidal suspension in water

\begin{tabular}{ccc}
\hline Species & Number density $\left[\mathbf{c m}^{-3}\right]$ & $\begin{array}{c}\text { Relative } \\
\text { percentage }\end{array}$ \\
\hline Ni-NiO & $8.38 \times 10^{10}$ & $75 \%$ \\
Air-Ni & $1.02 \times 10^{10}$ & $9 \%$ \\
NiO-Ni & $1.77 \times 10^{10}$ & $16 \%$ \\
\hline
\end{tabular}

As in the case of Ni NPs in n-heptane, hollow NPs are present in the water colloidal suspension. Its formation could be related to the laser induced bubbles. The optical breakdown induced by laser ablation in water, produces $\mathrm{H}_{2}$ gas as by-product from the following reaction

$$
\mathrm{Ni}+\mathrm{H}_{2} \mathrm{O} \rightarrow \mathrm{NiO}+\mathrm{H}_{2}
$$

Besides, $\mathrm{O}_{2}$ and $\mathrm{H}_{2}$ may also be generated in water at the focus of the laser beam. The gas content increases the stability of the bubbles, ${ }^{39}$ providing a larger probability for hollow particle formation. Since during fs laser ablation high temperatures and pressures are easily reached in the plasma plume and in the plasma-liquid interface ${ }^{42}$ during a time interval compatible with the fast oxidation kinetics at the nanoscale (about $100 \mathrm{~ns}$ ), it is possible to argue that, under our experimental conditions, the previous reaction may generate oxidized species in the form of $\mathrm{NiO}$ and $\mathrm{Ni}-\mathrm{NiO} \mathrm{NPs}$ in the colloidal suspension.

There is another process that must be considered. Medford et al. reported that when hollow oxidized Ni NPs in the size range $10 \mathrm{~nm}$ to $90 \mathrm{~nm}$ are exposed to a low content $\mathrm{H}_{2}$ atmosphere at $350{ }^{\circ} \mathrm{C}$, they undergo a reduction process (which depends on 
the initial size of the NPs) that gives rise to a metal shell around the particles. ${ }^{43}$ As stated above in equation (12), during fs laser ablation of $\mathrm{Ni}, \mathrm{H}_{2}$ is produced as a byproduct that, together with the high metal surface temperature obtained in the ablation process, allows the reduction of the formed NiO NPs, yielding a core-shell species of the type NiO-Ni in the colloidal suspension.

Figure 10a shows an AFM image of $30 \mu \mathrm{m} \times 25 \mu \mathrm{m}$ scanned area of isolated NPs in water colloidal suspension, dried on fresh mica surface. Height profiles scanned in lines 1 to 6 show NPs with typical external diameters of $15.9 \mathrm{~nm}, 86.3 \mathrm{~nm}, 6.4 \mathrm{~nm}$, $24.5 \mathrm{~nm}, 43.8 \mathrm{~nm}$ and $10 \mathrm{~nm}$ respectively, as indicated in Figure 10b. Line 3 shows the height profile of the mica background across the imaged area, which, as stated for Figure $7 \mathrm{~b}$, is much smaller than the measured NPs.

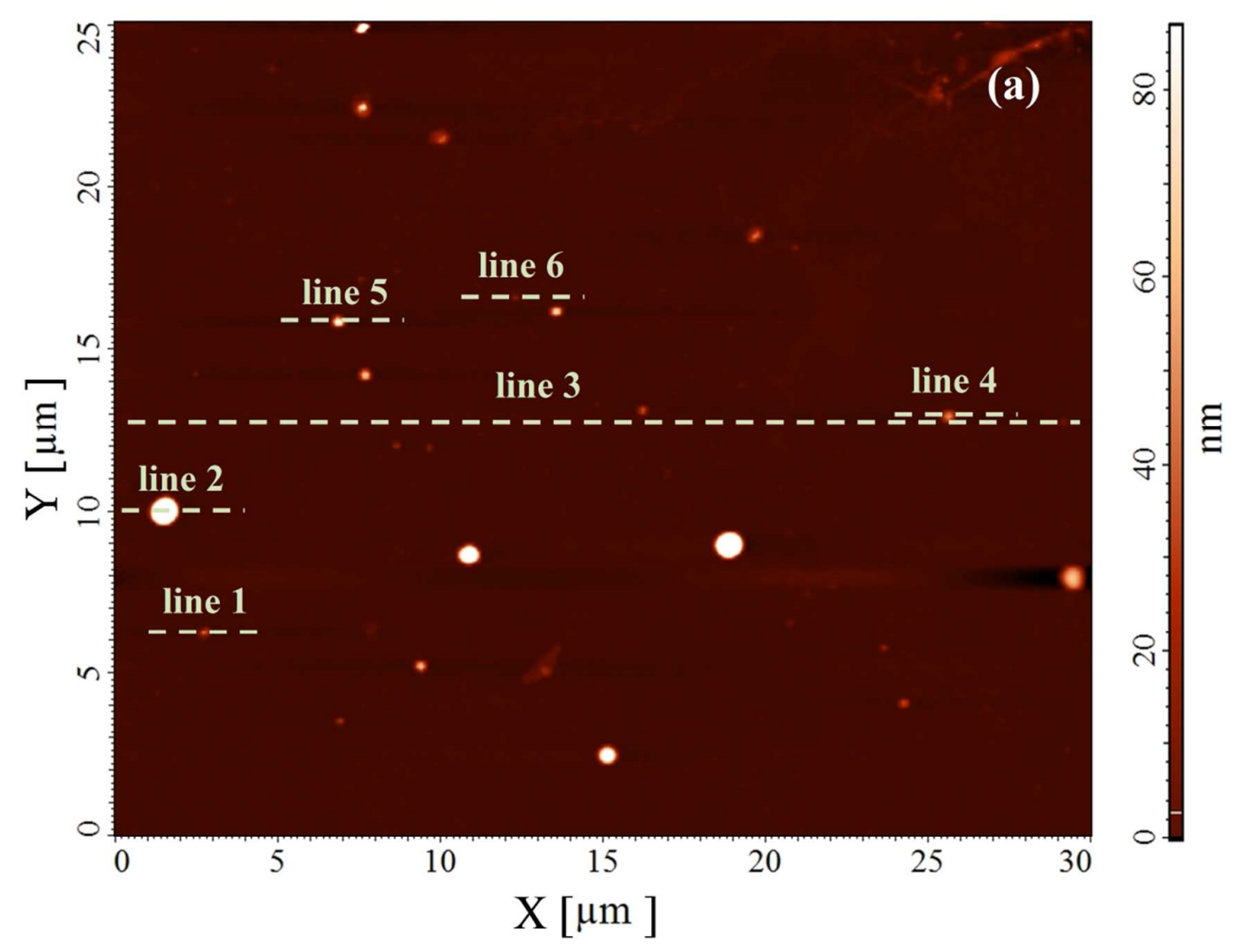




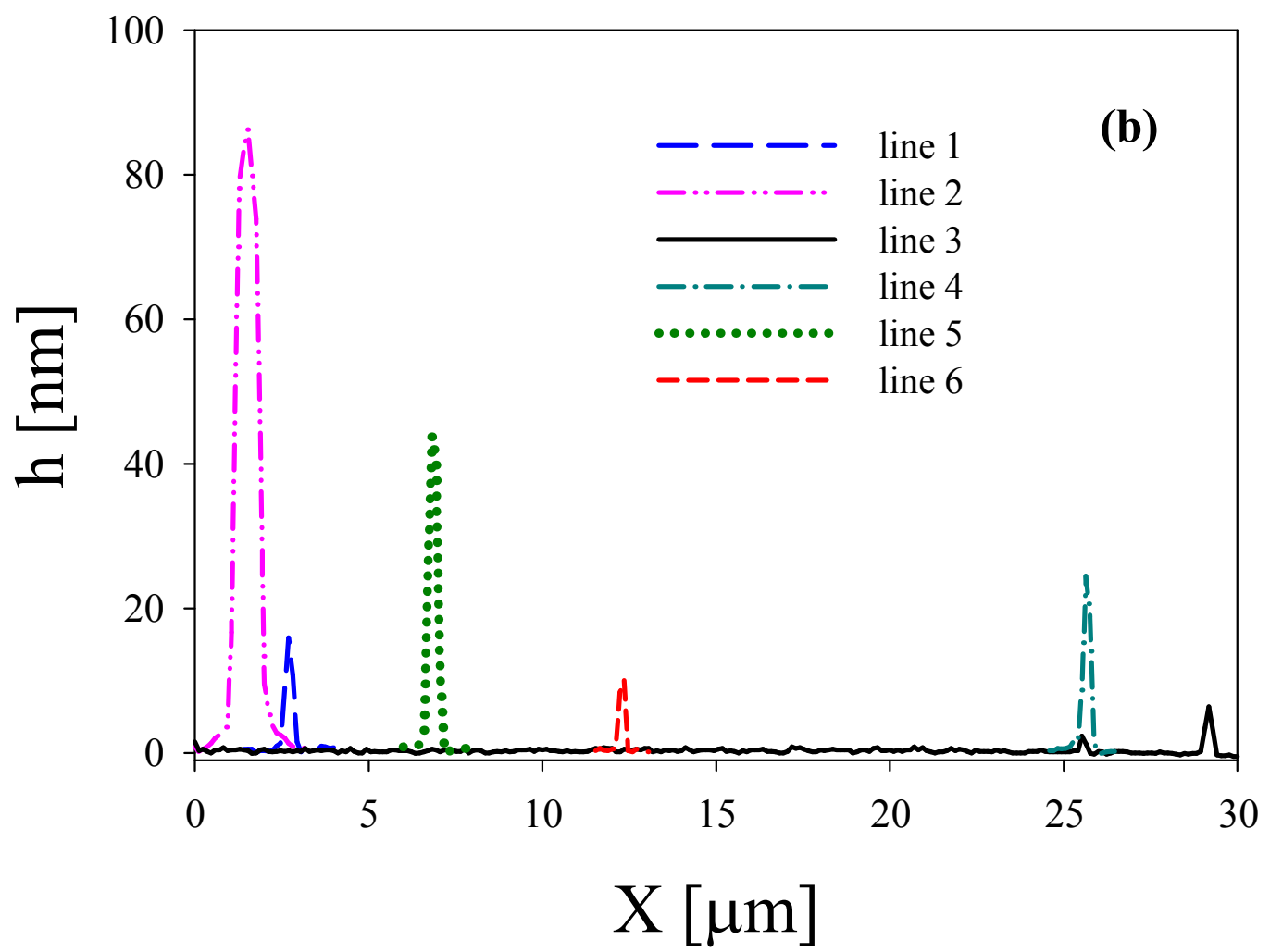

Figure 10. AFM size analysis of the obtained NPs after ablation of Ni target in water: (a) image of isolated NPs; (b) height profiles of selected lines 1 to 6 from panel (a).

Figure 11 shows TEM images of Ni colloidal suspension obtained by ablation in water. Panel (a) shows an isolated air-Ni core-shell NP. Panel (b) shows a bare core and core-shell NPs along with their respective sizes, placed in a different plane with respect to the other agglomerates of NPs. This is the reason why it is possible to observe other smaller NPs beyond the particle as the TEM electrons traverse across the sample. Panel (c) is a panoramic view where spherical particles showing different structures can be observed. Panel (d) shows the radius histogram corresponding to a statistics performed over several TEM images. Some isolated NPs with radius larger than $40 \mathrm{~nm}$, which are shown in these images for illustrative purposes, are not plotted on the histogram because they represent less than $1 \%$ of the observed number of particles. 
The results can be fitted by two log-normal size distributions which describe the most prominent features of the histogram. As in the case of n-heptane, there is a good agreement between TEM, AFM and OES results.

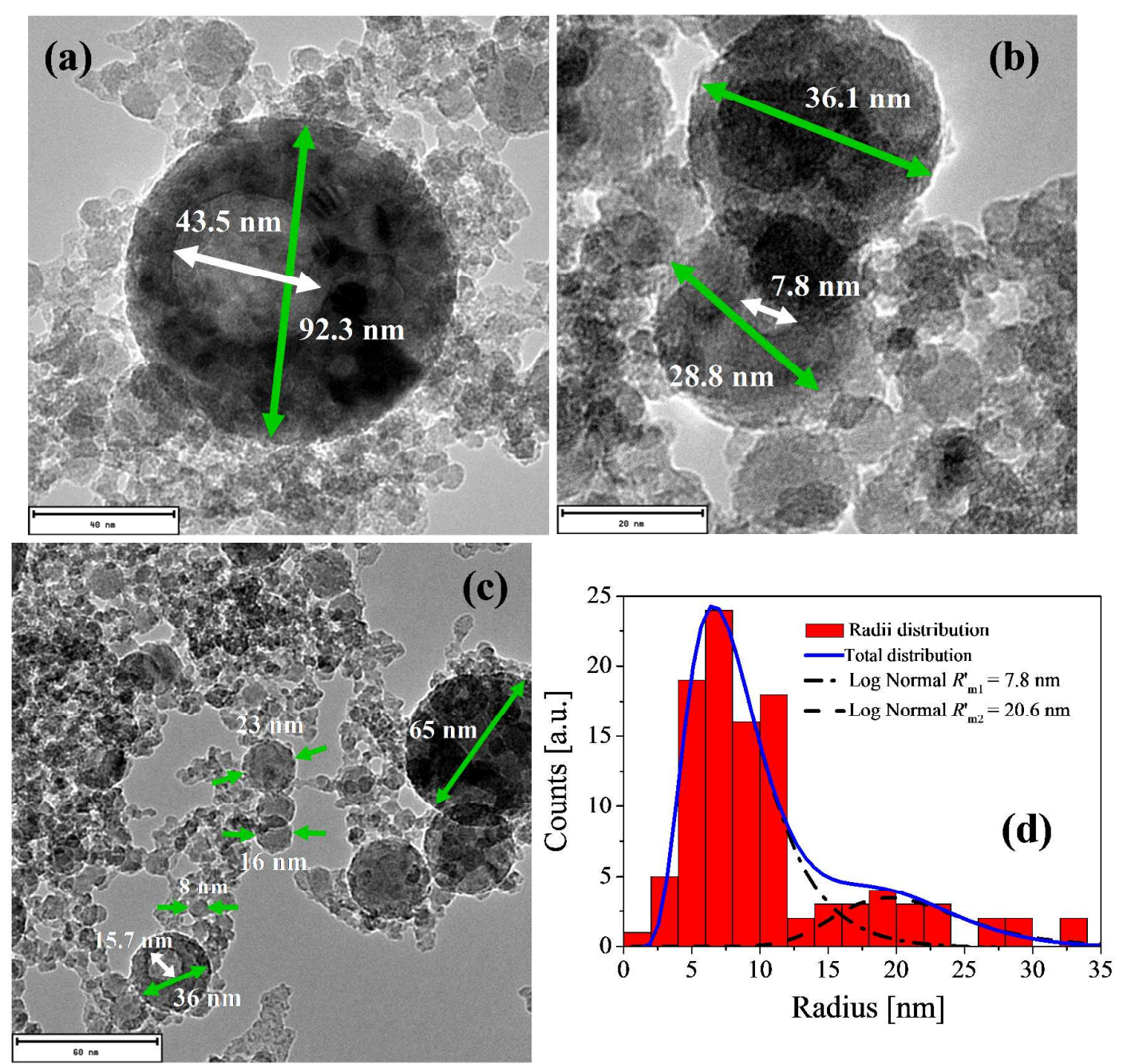

Figure 11. TEM image of Nps present in Ni colloidal suspension in water. (a) isolated single hollow Ni NP; (b) spherical bare core and core-shell NP. (c) panoramic view showing bare core and core-shell NPs and (d) radius histogram where two log-normal size distributions account for the most prominent features of the histogram.

TEM images of the NPs obtained by laser ablation are shown in Figure 12. Panel

(a) contains enlargements of two regions where NiO Bragg planes can be observed 
(Table 3). The NPs shown in Figure 12 are very similar to those reported by Medford et al. at the beginning of the reduction process by exposure to $\mathrm{H}_{2}{ }^{43}$

Electron diffraction patterns were measured on selected NPs for phase identification. A representative pattern is shown in Figure $12 \mathrm{~b}$, which is indexed with the reflection lines of NiO (Cubic, $F m \overline{3} m$, JCPDS \#75-0197) and Ni (Cubic, $F m \overline{3} m$, JCPDS \#04-0850). Electron diffraction rings are labeled according to Table 3, where the Miller indices $(h, k, l)$ and the interplanar distances (d) are indicated. No reflection characteristics of other structures appear.

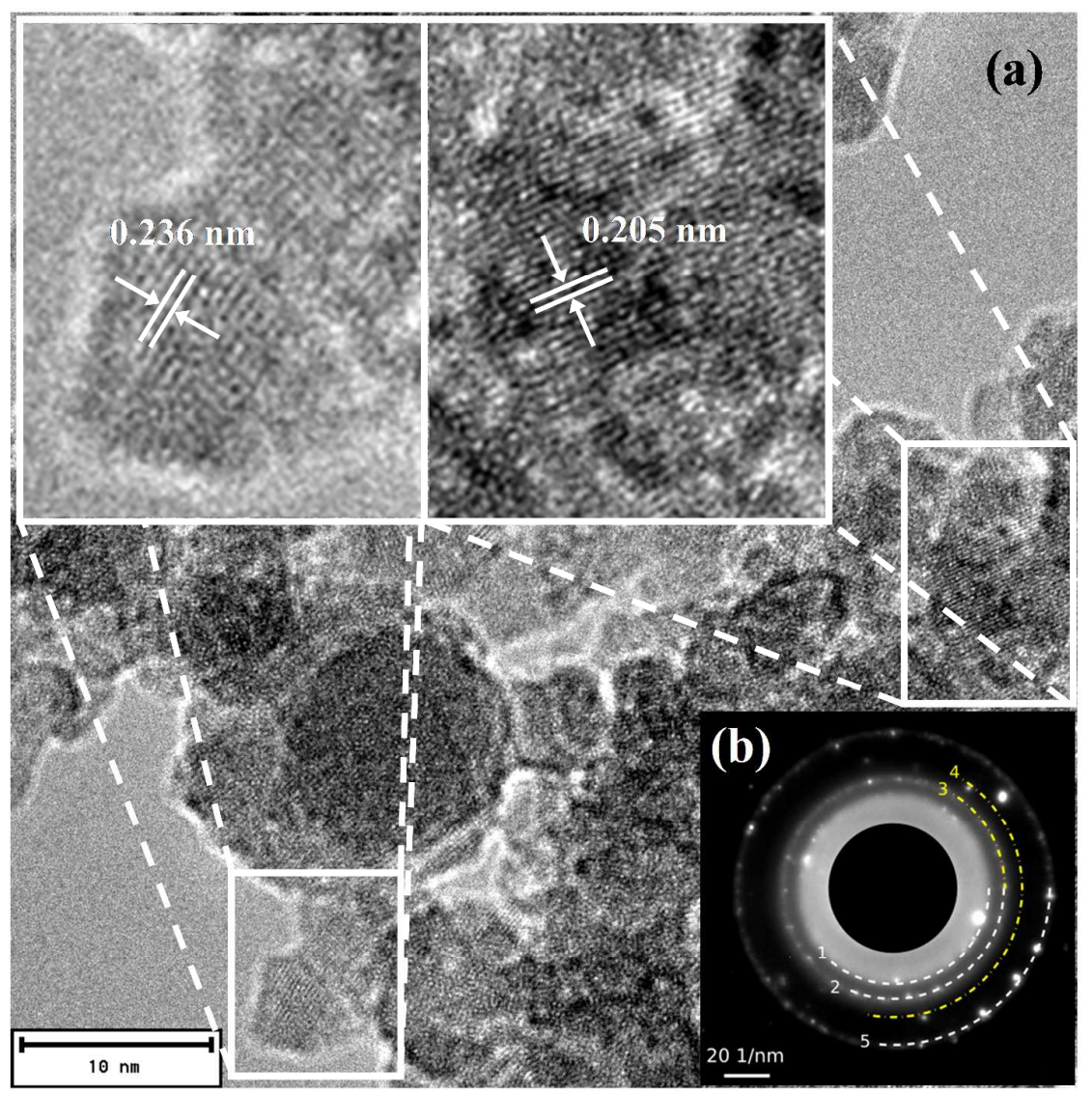

Figure 12. (a) TEM image of Ni NPs obtained by laser ablation in water; enlargements show typical regions where NiO Bragg planes can be observed, (b) Electron diffraction pattern indexed with the reflection lines of $\mathrm{NiO}$ (lines 1, 2 and 5) and $\mathrm{Ni}$ (lines 3 and 4) according to Table 3 . 
Table 3. Interplanar distances (d) and Miller indices $(h, k, l)$ for Ni (JCPDS \#04-0850) and NiO (JCPDS \#75-0197) used to index the electron diffraction pattern

\begin{tabular}{cccc}
\hline Ring & $\mathbf{d}_{\mathbf{N i}}(\mathbf{n m})$ & $\mathbf{d}_{\mathbf{N i O}}(\mathbf{n m})$ & $(\boldsymbol{h}, \boldsymbol{k}, \boldsymbol{l})$ \\
\hline 1 & & 0.2369 & $(1,1,1)$ \\
2 & & 0.2052 & $(2,0,0)$ \\
3 & 0.2034 & & $(1,1,1)$ \\
4 & 0.1762 & & $(2,0,0)$ \\
5 & & 0.1451 & $(2,2,0)$ \\
\hline
\end{tabular}

\section{CONCLUSIONS}

Colloidal Ni NPs were generated in $n$-heptane and water after fs pulsed laser ablation on solid target. The suspensions were analyzed using spectroscopic, microscopy and electron diffraction techniques to characterize their structure, configuration, composition and sizing. NPs resulted spherical in shape, with bare core or core-shell structure.

Fit of the experimental extinction spectra in each solvent using an iterative method based on Mie theory with appropriate size correction of the bulk complex dielectric function yielded the size distributions of bare core and core-shell NPs in the Ni colloidal suspension. For n-heptane, the total log-normal size distribution had a modal radius of $2.5 \mathrm{~nm}$ with a secondary maximum at $7 \mathrm{~nm}$ and $12 \mathrm{~nm}$. This 
distribution consists of two different structures: bare core and hollow Ni NPs. For water, the total log-normal size distribution had a modal radius of $4 \mathrm{~nm}$ with a secondary broad distribution centered at $20 \mathrm{~nm}$. In this case, only core-shell structures composed by hollow Ni, Ni-NiO and NiO-Ni NPs contribute to the total distribution. The formation of $\mathrm{NiO}-\mathrm{Ni}$ and hollow $\mathrm{Ni}$ species is reported for the first time in this paper.

In spite the number of particles observed in the AFM image is much smaller than that probed by OES (which is of about $10^{10}$ ), the AFM heights (diameters) histogram is in good agreement with the external radii distribution determined from the fit of the extinction spectrum. TEM analysis yielded spherical bare core, metal-metal oxide core-shell structures and hollow air-Ni NPs. This fact confirms the results obtained using Mie theory with the inclusion of core-shell and hollow particles for fitting the spectra. Since OES provides a non local analysis of the samples, as compared with TEM and AFM, we consider that OES yields statistically reliable results about size distribution and structures.

Electron diffraction patterns were measured on selected NPs for phase identification. The obtained representative pattern was indexed with the reflection lines of $\mathrm{NiO}$ and $\mathrm{Ni}$, thus also supporting the species distribution obtained after the optical absorbance spectrum fitting. The formation of Ni-oxide core-shell species may be explained by the generation of oxygen atoms during laser ablation in water and subsequent metal oxidation processes. Metal shell formation in NiO-Ni NP may arise due to exposure of $\mathrm{NiO} \mathrm{NP}$ to a low-content $\mathrm{H}_{2}$ environment due to laser photolysis. On the other hand, hollow Ni NPs may be produced during bubble formation in laser ablation. 


\section{ASSOCIATED CONTENT}

\section{Supporting Information}

Equations for theoretical calculations of extinction cross section, as well as full description of references 8 and 17, have been provided. This material is available free of charge via the Internet at http://pubs.acs.org.

\section{AUTHOR INFORMATION}

\section{Corresponding Author}

*Phone: +54 221 4840280/4715249 int. 213. Fax: +54 221 4712771. E-mail: lucias@ciop.unlp.edu.ar.

\section{Notes}

The authors declare no competing financial interest.

\section{ACKNOWLEDGMENTS}

This work was granted by PIP 0394 and PIP 0720 of CONICET, PME200600018 of ANPCyT, grant 11/I151 of Facultad de Ingeniería Universidad Nacional de La Plata and grant 11/X680 of Facultad de Ciencias Exactas Universidad Nacional de La Plata, Argentina. We thank C2NANO - Brazilian Nanotechnology National Laboratory (LNNano) at Centro Nacional de Pesquisa em Energia e Materiais (CNPEM) / MCT (\#14825 and 14827) and Research Proposal TEM-16976 for the use of TEM. AFM was carried out at LFAyM of Instituto de Física La Plata (IFLP - CONICET). We acknowledge Dr. Francisco Sánchez from Instituto de Física La Plata (IFLP CONICET) for fruitful discussions on magnetic nanoparticles. 
D. C. Schinca is Member of Comisión de Investigaciones Científicas de la Provincia de Buenos Aires (CIC). L. B. Scaffardi, M. B. Fernández van Raap, P. Mendoza Zélis and J. M. J. Santillán are researchers of CONICET. D. Muraca is Member of Instituto de Física “Gleb Wataghin” (IFGW), Campinas, Brazil. D. Muñetón Arboleda and L. J. Mendoza Herrera are PhD fellows of CONICET, Argentina.

\section{REFERENCES}

(1) Bönnemann, H.; Richards, R. M. Nanoscopic Metal Particles - Synthetic Methods and Potential Applications. Eur. J. Inorg. Chem. 2001, 10, 2455-2480.

(2) Chen, C. C.; Herhold, A. B.; Johnson, C. S.; Alivisatos, A. P. Size Dependence of Structural Metastability in Semiconductor Nanocrystals. Science 1997, 276, 398-401.

(3) Puntes, V. F.; Krishnan, K. M.; Alivisatos, A. P. Colloidal Nanocrystal Shape and Size Control: The Case of Cobalt. Science 2001, 291, 2115-2117.

(4) Andrade, A. L.; Valente, M. A.; Ferreira, J. M. F.; Fabris, J. D. Preparation of SizeControlled Nanoparticles of Magnetite. J. Magn. Magn. Mater. 2012, 324, 1753-1757.

(5) Hou, Y.; Gao, S. Monodisperse Nickel Nanoparticles Prepared from a Monosurfactant System and their Magnetic Properties. J. Mater. Chem. 2003, 13, 15101512.

(6) Sun S.; Murray, C. B.; Weller, D.; Folks, L.; Moser, A. Monodisperse FePt Nanoparticles and Ferromagnetic FePt Nanocrystal Superlattices. Science 2000, 287, 1989-1992.

(7) Beecroft, L. L.; Ober, C. K. Nanocomposite Materials for Optical Applications. Chem. Mater. 1997, 9, 1302-1317.

(8) Park, J.; Kang, E.; Son, S. U.; Park, H. M.; Lee, M. K.; Kim, J.; Kim, K. W.; Noh, H. J.; Park, J. H.; Bae, C.; et al. Monodisperse Nanoparticles of Ni and NiO: Synthesis, 
Characterization, Self-Assembled Superlattices, and Catalytic Applications in the Suzuki Coupling Reaction. Adv. Mater. 2005, 17, 429-434.

(9) Xu, R.; Xie, T.; Zhao, Y.; Li, Y. Quasi-Homogeneous Catalytic Hydrogenation over Monodisperse Nickel and Cobalt Nanoparticles. Nanotechnology 2007, 18, 055602055605.

(10) Bai, L.; Yuan, F.; Tang, Q. Synthesis of Nickel Nanoparticles with Uniform Size via a Modified Hydrazine Reduction Route. Mater. Lett. 2008, 62, 2267-2270.

(11) Wessells, C. D.; Peddada, S. V.; Huggins, R. A.; Cui, Y. Nickel Hexacyanoferrate Nanoparticle Electrodes for Aqueous Sodium and Potassium Ion Batteries. Nano Lett. 2011, 11, 5421-5425.

(12) Couto, G. G.; Klein, J. J.; Schreiner, W. H.; Mosca, D. H.; de Oliveira, A. J. A.; Zarbin, A. J. G. Nickel Nanoparticles Obtained by a Modified Polyol Process: Synthesis, Characterization, and Magnetic Properties. J. Colloid Interf. Sci. 2007, 311, $461-468$.

(13) Ramírez-Meneses, E.; Betancourt, I.; Morales, F.; Montiel-Palma, V.; VillanuevaAlvarado, C. C.; Hernández-Rojas, M. E. Superparamagnetic Nickel Nanoparticles Obtained by an Organometallic Approach. J. Nanopart. Res. 2011, 13, 365-374.

(14) Ni, X.; Zhao, Q.; Zheng, H.; Li, B.; Song, J.; Zhang, D.; Zhang, X. A Novel Chemical Reduction Route Towards the Synthesis of Crystalline Nickel Nanoflowers from a Mixed Source. Eur. J. Inorg. Chem. 2005, 4788-4793.

(15) Cheng, J.; Zhang, X.; Ye, Y. Synthesis of Nickel Nanoparticles and Carbon Encapsulated Nickel Nanoparticles Supported on Carbon Nanotubes. J. Solid State Chem. 2006, 179, 91-95.

(16) Murugadoss, G.; Rajesh Kumar, M. Synthesis and Optical Properties of Monodispersed $\mathrm{Ni}^{2+}$-Doped ZnS Nanoparticles. Appl. Nanosci. 2014, 4, 67-75. 
(17) Rodríguez-Llamazares, S.; Merchán, J.; Olmedo, I.; Marambio, H. P.; Muñoz, J. P.; Jara, P.; Sturm, J. C.; Chornik, B.; Peña, O.; Yutronic, N.; et al. Ni/Ni Oxides Nanoparticles with Potential Biomedical Applications Obtained by Displacement of a Nickel-Organometallic Complex. J. Nanosci. Nanotechnol. 2008, 8, 3820-3827.

(18) Liu, B.; Hu, Z.; Che, Y.; Chen, Y.; Pan, X. Nanoparticle Generation in Ultrafast Pulsed Laser Ablation of Nickel. Appl. Phys. Lett. 2007, 90, 044103-3.

(19) Amoruso, S.; Bruzzese, R.; Wang, X.; Nedialkov, N. N.; Atanasov, P. A. Femtosecond Laser Ablation of Nickel in Vacuum. J. Phys. D: Appl. Phys. 2007, 40, 331-340.

(20) Dudoitis, V.; Ulevičius, V.; Račiukaitis, G.; Špirkauskaitè, N.; Plauškaitė, K. Generation of Metal Nanoparticles by Laser Ablation. Lithuanian J. Phys. 2011, 51, $248-259$.

(21) Jaleh, B.; Torkamany, M. J.; Golbedaghi, R.; Noroozi, M.; Habibi, S.; Samavat, F.; Jaberian Hamedan, V.; Albeheshti, L. Preparation of Nickel Nanoparticles via Laser Ablation in Liquid and Simultaneously Spectroscopy. Adv. Mater. Res. 2012, 403-408, $4440-4444$.

(22) Jung, H. J.; Choi, M. Y. Specific Solvent Produces Specific Phase Ni Nanoparticles: A Pulsed Laser Ablation in Solvents. J. Phys. Chem. C 2014, 118, $14647-14654$.

(23) Musaev, O. R.; Yan, J.; Dusevich, V.; Wrobel, J. M.; Kruger, M. B. Ni Nanoparticles Fabricated by Laser Ablation in Water. Appl. Phys. A 2014, 116, 735739.

(24) Bohren, C. F.; Huffmanm, D. R. Absorption and Scattering of Light by Small Particles; Wiley: New York, 1998. 
(25) Rosensweig, R. E. Heating Magnetic Fluid with Alternating Magnetic Field. J. Magn. Magn. Mat. 2002, 252, 370-374.

(26) Goya, G. F.; Fonseca, F. C.; Jardim, R. F.; Muccillo, R.; Carreño, N. L. V.; Longo, E.; Leite, E. R. Magnetic Dynamics of Single Domain Ni Nanoparticles. J. Appl. Phys. 2003, 93, 6531 .

(27) Kreibig, U.; Vollmer, M. Optical Properties of Metal Clusters; Springer: Berlin, 1995.

(28) Scaffardi, L. B.; Tocho, J. O. Size Dependence of Refractive Index of Gold Nanoparticles. Nanotechnology 2006, 17, 1309-1315.

(29) Mendoza Herrera, L. J.; Muñetón Arboleda, D.; Schinca, D. C.; Scaffardi, L. B. Determination of Plasma Frequency, Damping Constant, and Size Distribution from the Complex Dielectric Function of Noble Metal Nanoparticles. J. Appl. Phys. 2014, 116, 233105-8.

(30) Ordal, M. A.; Bell, R. J.; Alexander, R. W. Jr.; Long, L. L.; Querry, M. R. Optical Properties of $\mathrm{Au}, \mathrm{Ni}$, and $\mathrm{Pb}$ at Submillimeter Wavelengths. Appl. Opt. 1987, 26, 744752.

(31) Rakić, A. D.; Djurišic, A. B.; Elazar, J. M.; Majewski, M. L. Optical Properties of Metallic Films for Vertical-Cavity Optoelectronic Devices. Appl. Opt. 1998, 37, 52715283.

(32) Petrovykh, D. Y.; Altmann, K. N.; Hochst, H.; Laubscher, M.; Maat, S.; Mankey, G. J.; Himpse, F. J. Spin-dependent Band Structure, Fermi Surface, and Carrier Lifetime of Permalloy. Appl. Phys. Lett. 1998, 73, 3459-3461.

(33) Khan, S. Z.; Yuan, Y.; Abdolvand, A.; Schmidt, M.; Crouse, P.; Li, L.; Liu, Z.; Sharp, M.; Watkins, K. G. Generation and Characterization of NiO Nanoparticles by 
Continuous Wave Fiber Laser Ablation in Liquid. J. Nanopart. Res. 2009, 11, 14211427.

(34) Prodan, E.; Norlander, P. Structural Tunability of the Plasmon Resonances in Metallic Nanoshells. Nano Lett. 2003, 3, 543-547.

(35) Mahmoud, S. A.; Alshomer, S.; Tarawnh, M. A. Structural and Optical Dispersion Characterization of Sprayed Nickel Oxide Thin Films. J. Modern Phys. 2011, 2, 11781186.

(36) Desarkar, H. S.; Kumbhakar, P.; Mitra, A. K. One-step Synthesis of Zn/ZnO Hollow Nanoparticles by the Laser Ablation in Liquid Technique. Laser Phys. Lett. 2013, 10, 055903-8.

(37) Yan, Z.; Bao, R.; Chrisey, D. B. Excimer Laser Ablation of a Pt Target in Water: The Observation of Hollow Particles. Nanotechnology 2010, 21, 145609-8.

(38) Yan, Z.; Bao, R.; Huang, Y.; Chrisey, D. B. Hollow Particles Formed on LaserInduced Bubbles by Excimer Laser Ablation of Al in Liquid. J. Phys. Chem. C 2010, $114,11370-11374$.

(39) Yan, Z.; Bao, R.; Wright, R. N.; Chrisey, D. B. Hollow nanoparticle generation on Laser-Induced Cavitation Bubbles via Bubble Interface Pinning. Appl. Phys. Lett. 2010, 97, 124106-3.

(40) Niu, K. Y.; Yang, J.; Kulinich, S. A.; Sun, J.; Du, X. W. Hollow Nanoparticles of Metal Oxides and Sulfides: Fast Preparation via Laser Ablation in Liquid. Langmuir 2010, 26, 16652-16657.

(41) Santillán, J. M. J.; Videla, F. A.; Fernández van Raap, M. B.; Muraca, D.; Scaffardi, L. B.; Schinca, D. C. Influence of Size-Corrected Bound-Electron Contribution on Nanometric Silver Dielectric Function. Sizing through Optical Extinction Spectroscopy. J. Phys. D: Appl. Phys. 2013, 46, 435301-10. 
(42) Nath, A.; Khare, A. Size Induced Structural Modifications in Copper Oxide Nanoparticles Synthesized via Laser Ablation in Liquids. J. Appl. Phys. 2011, 110, 043111.

(43) Medford, J. A.; Johnston-Peck, A. C.; Tracy, J. B. Nanostructural Transformations During the Reduction of Hollow and Porous Nickel Oxide Nanoparticles. Nanoscale 2013, 5, 155-159. 


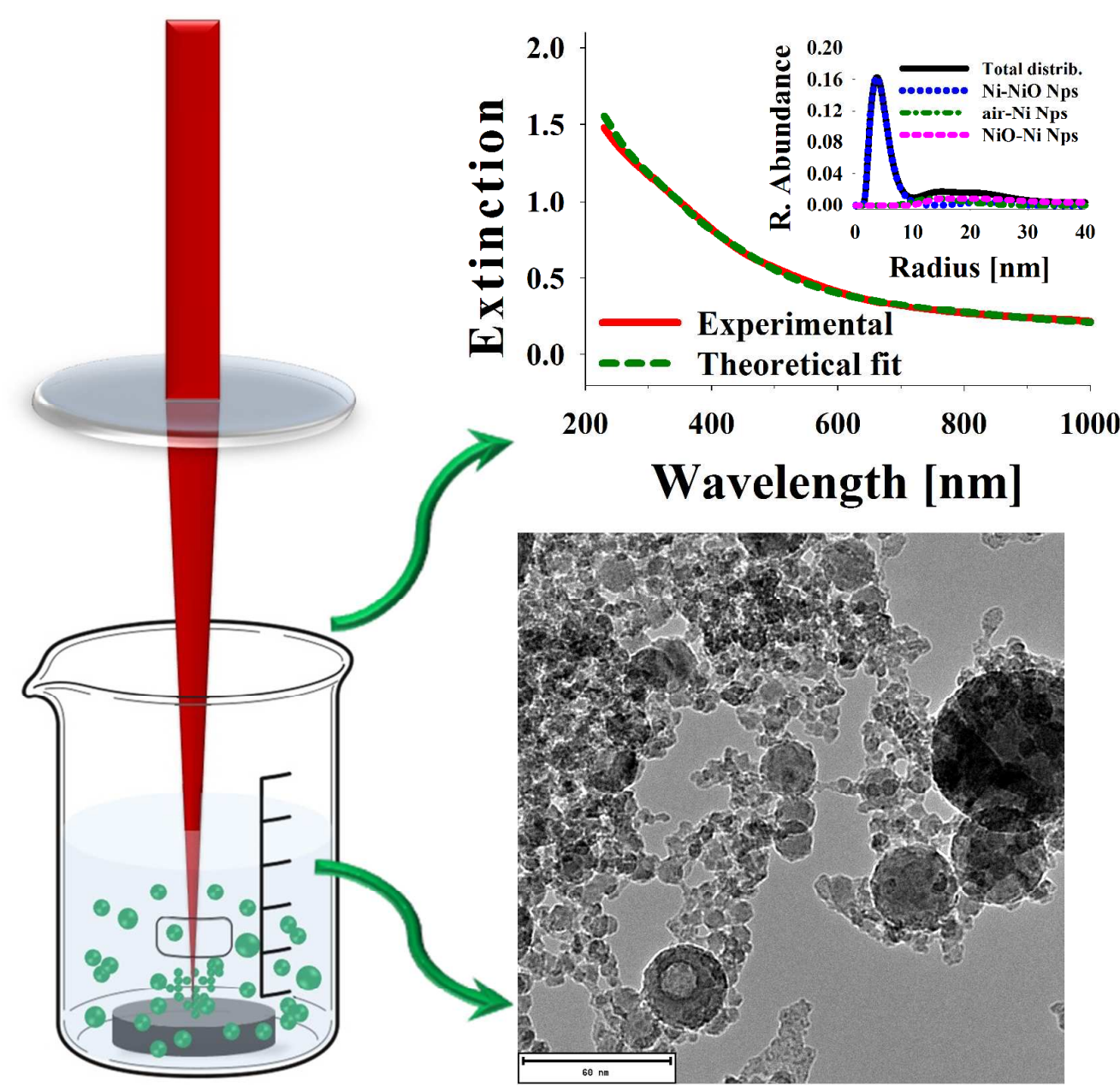

